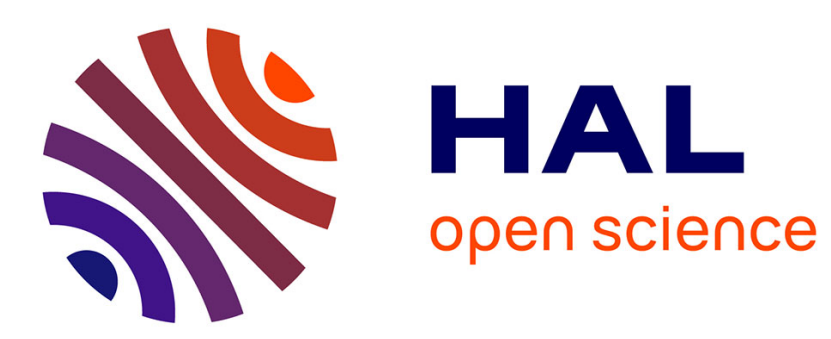

\title{
A Unified Methodology to Evaluate the Radiated Noise Due to Turbulent Boundary Layer Flows
}

\author{
Sylvain Morilhat, François Chedevergne, Frank Simon
}

\section{To cite this version:}

Sylvain Morilhat, François Chedevergne, Frank Simon. A Unified Methodology to Evaluate the Radiated Noise Due to Turbulent Boundary Layer Flows. Journal of Fluids Engineering, 2018, 141 (6), pp.061201-1 - 061201-11. 10.1115/1.4041611 . hal-01999610

\section{HAL Id: hal-01999610 https://hal.science/hal-01999610}

Submitted on 30 Jan 2019

HAL is a multi-disciplinary open access archive for the deposit and dissemination of scientific research documents, whether they are published or not. The documents may come from teaching and research institutions in France or abroad, or from public or private research centers.
L'archive ouverte pluridisciplinaire HAL, est destinée au dépôt et à la diffusion de documents scientifiques de niveau recherche, publiés ou non, émanant des établissements d'enseignement et de recherche français ou étrangers, des laboratoires publics ou privés. 


\title{
A unified methodology to evaluate the radiated noise due to turbulent boundary layer flows
}

\author{
Sylvain Morilhat \\ ONERA-DMPE \\ 2 Avenue E. Belin \\ 31000 Toulouse \\ France \\ Email: sylvain.morilhat@onera.fr
}

\author{
Francois Chedevergne \\ ONERA-DMPE \\ 2 Avenue E. Belin \\ 31000 Toulouse \\ France \\ Email: francois.chedevergne@onera.fr
}

\author{
Frank Simon \\ ONERA-DMPE \\ 2 Avenue E. Belin \\ 31000 Toulouse \\ France \\ Email:frank.simon@onera.fr
}

\begin{abstract}
For vibro-acoustic applications, a turbulent wall pressure fluctuations model was derived. The model is based on the resolution of the Poisson equation. The pressure is characterized in time and space through its spectrum in the frequency wave-number domain. The developed model follows trends commonly observed using Corcos model in a large frequency range but also shows new behaviours for low and high frequencies. The radiated noise due to turbulent wall pressure fluctuations is then computed in accordance with the form of the turbulent wall pressure spectrum. A specific computational methodology is proposed to perform the calculation without introducing limiting hypothesis on the radiated impedance.
\end{abstract}

\section{INTRODUCTION}

The internal noise perceived in helicopter cockpits is caused by several sources. Among them, flow induced noise is becoming a growing area of interest. For decades, it was considered negligible compared to noise sources generated by the engines which were predominant. Thanks to the efforts made to reduce those sources of noises, the flow induced noise must now be considered in order to improve passengers comfort in flight.

The unsteady turbulent wall pressure (TWP) due to boundary layers developing along external surfaces, generate a vibratory motion of walls creating radiated noise that propagates inside aircraft. Modelling the whole scenario requires associating turbulence and vibro-acoustic models, related to each other through the fluctuating wall pressure. The stochastic nature of turbulence makes the TWP hardly expressible in the physical domain $p(x, t)$. It is generally described with appropriate functions such as inter-correlations in space and time $S_{p p}(r, \tau)$, or similarly using Fourier transforms as spectra in the wavenumber and frequency domain $\Phi_{p p}(k, \omega)$. In this context, modelling the turbulence fluctuations at the wall reduces down to modelling the spectrum $\Phi_{p p}(k, \omega)$. From this spectrum, vibro-acoustics tools are applied to compute the radiated noise. From a theoretical point of view, these two tasks can be performed separately. But practically the form of $\Phi_{p p}(k, \omega)$ greatly affects the numerical implementation of the computation of the radiated noise. For this reason, it is interesting to have a complete modelling accounting for both turbulence and vibro-acoustics issues available in a unified context. The goal of this article is twofold. First, a more realistic model for the spectrum $\Phi_{p p}(k, \omega)$, compared to those available in the literature, is proposed. Second, standard vibro-acoustics tools are revisited to make them compatible with the proposed form of the $\Phi_{p p}(k, \omega)$ model. The whole set forms a methodological framework for computing the radiated noise due to turbulent boundary layers. 
To model $\Phi_{p p}(k, \omega)$ a commonly used approach is to split the spectrum $\Phi_{p p}(k, \omega) \approx \phi_{p p}(\omega) \times \varphi_{p p}(k, \omega)$ into a form function $\varphi_{p p}(k, \omega)$ and an amplitude function $\phi_{p p}(\omega)$ such that $(2 \pi)^{-2} \int \varphi_{p p}(k, \omega) \mathrm{d} k=1$. Concerning $\phi_{p p}(\omega)$, models can rely on experimental data since measurements only require single-point information. On the theoretical side, only asymptotic behaviours were obtained as discussed by Panton[1], Blake [2] and Bradshaw [3] : $\phi_{p p}(\omega) \underset{\omega \rightarrow 0}{\propto} \omega^{2}$ and $\phi_{p p}(\omega) \underset{\omega \rightarrow \infty}{\propto} \omega^{-5}$. As discussed by [4] based on empirical data, different characteristic scales has to be used for high and low frequencies. The outer variables suit low frequencies while wall variables suit high frequencies The overlapping region may be approximated using $\phi_{p p}(\omega) \propto \omega^{-1}$ and both scaling can be used. Slopes in $\omega^{2}$ and $\omega^{-5}$ were observed experimentally by Farabee and Casarella[5] but $\omega^{-1}$ matching remains questionable. Instead, slopes between $\omega^{-0.7}$ and $\omega^{-0.8}$ were observed by Mcgrath and Simpson[6], Blake[7] and Goody and Simpson[8]. Even though asymptotic behaviours were found for $\phi_{p p}$, there is no theoretical model for $\phi_{p p}$ covering the whole spectral domain. Most of the models available in the literature are thus based on empirical correlations. Their forms are chosen such that the asymptotic behaviours are recovered and reproduce experimental data. These models rely on turbulent boundary layer (TBL) parameters such as free-stream velocity $U_{e}$, boundary layer thickness $\delta$, momentum thickness $\theta$, friction velocity $u_{\tau}$ or pressure gradient. Correlations for the zero pressure gradient boundary layers were proposed by Efimstov[9, 10], Goody[11] and Catlett et al. [12] while Rozenberg[13], Hu and Herr[14], Hu[15] and Kamruzzaman et al.[16] presented correlations for adverse pressure gradient cases. Zero pressure gradient models have been compared by Hwang et al. [17] while adverse pressure gradient models have been compared by Lee et al.[18].

Models for the spatial part $\varphi_{p p}(k, \omega)$ also rely on empirical considerations and result in correlations made to reproduce experimental data. Most of the models used in the literature are inspired by Corcos[19, 20] works. Based on experimental observations, a model for the spatial correlation of the TWP was built with an oscillatory exponential decay in the longitudinal direction $x$ and an exponentially decaying form in the transverse direction $z$ :

$$
\begin{aligned}
S_{p p}(x, z, x+\delta x, z+\delta z, \omega) & =\phi_{p p}(\omega) \exp \left(-\alpha\left|\frac{\omega \delta x}{U_{c}}\right|\right) \exp \left(-\beta\left|\frac{\omega \delta z}{U_{c}}\right|+j \frac{\omega \delta x}{U_{c}}\right) \\
U_{c} & =0.7 U_{e}(x) \\
\alpha & =0.116 \\
\beta & =0.7
\end{aligned}
$$

Corcos model only provides information on the spatial behaviour as the correlation $S_{p p}$ depends on an unknown function $\phi_{p p}(\omega)$ of the frequency $\omega$. The spectrum $\Phi_{p p}(k, \omega)$ associated to this correlation is then obtained through a Fourier transform in space using the product of two Cauchy distributions in each direction:

$$
\begin{aligned}
\Phi_{p p}\left(k_{x}, k_{z}, \omega\right) & =\phi_{p p}(\omega) \frac{2 \pi \alpha}{k_{c}\left[\alpha^{2}+\left(k_{x} / k_{c}+1\right)^{2}\right]} \frac{2 \pi \beta}{k_{c}\left[\beta^{2}+\left(k_{z} / k_{c}\right)^{2}\right]} \\
k_{c} & =\omega / U_{c}
\end{aligned}
$$

Corcos work was improved by several authors proposing new values for parameters $\alpha, \beta, U_{c} / U_{e}$. For instance, Efimtsov $[9,10]$ accounted for compressibility effects and the boundary layer thickness to compute values of $\alpha$ and $\beta$.

To avoid resorting to the splitting introduced above and to have a more realistic modelling of $\Phi_{p p}(k, \omega)$, the Poisson equation that governs the fluctuations of the turbulent pressure can be directly considered. The idea of solving Poisson's equation with Green's functions dates back to Kraichnan[21] in 1956. However, the determination of $\Phi_{p p}$ requires the knowledge of the mean profiles in the boundary layer and the spectra of the fluctuating velocities. As these quantities were not available for Kraichnan, only the simplified case of a constant shear stress profile was considered and its study was limited to determine orders of magnitude. Moreover, the model requires the computation of improper integrals that cannot be solved analytically. About twenty years later, when these integrals could be numerically evaluated, Panton and Linebarger[1] computed spatial and frequency spectra. The TBL profile was described through semi-analytical models. Most of the modern $\Phi_{p p}$ models rely on RANS calculations to get the TBL profiles. Two types of approaches may be distinguished. On one side, works following the spectral approach of Kraichnan, based on a wave-number-frequency resolution, may be gathered such as works by Lee et al.[22, 23], Remmler et al.[24], Parchen[25] latterly extended by Bertagnolio et al.[26, 27]. All these models give only the frequency spectrum $\phi_{p p}(\omega)$ and not the complete $\Phi_{p p}(k, \omega)$ spectrum. On the other side, works based on a space-time resolution of Poisson's equation such as Peltier et al. [28] and Slama et al.[29] result in an expression for the correlation $S_{p p}(r, \tau)$. The spectra $\phi_{p p}(\omega)$ and $\Phi_{p p}(k, \omega)$ are deduced through Fourier transforms but they must be performed numerically as no analytical formulations are available in these studies. 
As complete vibro-acoustics modelling requires $\Phi_{p p}(k, \omega)$ and not only $\phi_{p p}(\omega)$, models based on the resolution of Poisson's equation available in the literature are not completely suitable in the present context. Using $S_{p p}(r, \tau)$ is theoretically possible but leads to delicate numerical issues and very large computational costs. Recently, Kraichnan's approach was revisited by Lysak[30] for pipe flows. Aupoix[31] extended the approach to any boundary layer flow computed using a RANS solver. One of the objectives of the present work is to improve this approach to obtain the whole spectrum $\Phi_{p p}(k, \omega)$ in the wavenumber and frequency domain from the resolution of Poisson's equation. The resulting spectrum will then serve as an input for vibro-acoustics computations in order to evaluate the radiated noise.

Several studies[32-36] have already address the question of computing the radiated noise caused by turbulent boundary layers. The radiated noise is characterized by the acoustic intensity integrated over the whole vibrating surface. For that purpose, the TWP spectrum is modelled using Corcos formulation (2) and is assumed to behave as a combination of planar waves allowing to resort to Fourier transform for computations. This methodology was initiated in the 70's[37] and further extended by Hwang and Maidanik[38], Graham[33] and Maury et al.[39]. In the specific case of a rectangular plate simply supported on its edges, analytical expressions were obtained by Hwang and Maidanik[38] for the acoustic intensity. Such analytical solutions are no longer achievable when other structures are considered or when different forms for TWP spectra $\Phi_{p p}(k, \omega)$ are used.

In the present context, where the TWP spectrum is no longer described using Corcos model, the standard methodology for the computation of the radiated noise must be revisited. Starting from the definition of the acoustic intensity, the main difficulty lies in the calculation of the two-dimensional improper integrals over the wave-number domain. The retained approach for the TWP computation does not permit to separate the longitudinal and transversal contributions. To compute the acoustic intensity, a new methodology is proposed, based on a numerical evaluation of the improper integrals and a efficient procedure of selection of the main contributions.

The paper is organized as follows: the first section is dedicated to the model of the TWP spectrum. The original model of Aupoix [31] is recap and an extension is formulated to access the whole spectrum $\Phi_{p p}(k, \omega)$. The model is then compared to Corcos approach. The second section is devoted to the coupling with the vibrating structure. The methodology allowing the evaluation of the radiated noise is detailed and a computational algorithm is presented.

\section{Turbulence modelling}

This section concentrates on the modelling of term $\Phi_{p p}(k, \omega)$. In this study, this term relies on studies by Lysak[30] and Aupoix[31]. The basis of these works is briefly summarized in the following sub-section.

\subsection{Lysak and Aupoix's model}

The main idea of Lysak and Aupoix's approach is to solve the following Poisson's equation in order to access the TWP spectrum $\Phi_{p p}(k, \omega)$ :

$$
\nabla^{2} p=-\rho \frac{\partial^{2}}{\partial x_{i} \partial x_{j}}\left(2 U_{i} u_{j}+u_{i} u_{j}-\overline{u_{i} u_{j}}\right)
$$

The source terms correspond to:

. $-2 \rho \frac{\partial^{2}}{\partial x_{i} \partial x_{j}} U_{i} u_{j}$ which reflects the interactions between the turbulence and the mean flow (TM),

- $-\rho \frac{\partial^{2}}{\partial x_{i} \partial x_{j}}\left(u_{i} u_{j}-\overline{u_{i} u_{j}}\right)$ which accounts for the turbulent-turbulent (TT) interactions.

First, Lysak and Aupoix only consider the linear term $-2 \rho \frac{\partial^{2}}{\partial x_{i} \partial x_{j}} U_{i} u_{j}$ of Eq.(3) and more precisely only $-2 \rho \frac{\mathrm{d} U}{\mathrm{~d} y} \frac{\mathrm{d} v}{\mathrm{~d} x}$ is retained in a two-dimensional boundary layer context. They neglect the TT interactions. This assumption is questionable under some circumstances but there are no existing solutions to deal with the TT term when solving the Poisson equation in the spectral domain. In the approach initiated by Peltier et al.[28] and recently extended by Slamma et al.[29] that solves the Poisson equation in the physical space, both TM and TT terms are considered. As $S_{p p}$ is a second-order tensor, its computation through Eq.(3) requires velocity tensors of order two for TM terms and three and four for TT terms. Assuming that the fluctuating velocity fields satisfy the quasi-normality hypothesis, the third-order tensor is zero and the fourth-order tensor can be expressed using the second-order tensor just as for the TM term.

Second, the Poisson equation is solved using Green's functions. Then, the velocity fluctuations are modelled using an isotropic and homogeneous von Kàrmàn spectrum for the spatial part and a Dirac distribution using Taylor's frozen turbulence hypothesis for the frequency part. These two choices are discussed in Lysak[40]. The spectrum $\Phi_{v v}(k, \omega)$ reads 


$$
\begin{aligned}
\Phi_{v v}(k, \omega) & =(2 \pi)^{3} \frac{3}{8 \pi} \frac{\left\langle v^{2}\right\rangle}{k_{e}^{3}} \frac{\left(k_{x}^{2}+k_{z}^{2}\right)}{k_{e}^{2}}\left[1+|k|^{2} / k_{e}^{2}\right]^{-17 / 6} \\
& \times 2 \pi \delta\left(\omega+k_{x} U\right) \\
\left\langle v^{2}\right\rangle & =\mathbb{E}\left(v^{2}\right) \\
k_{e} & \text { wave number carrying the maximum of energy }
\end{aligned}
$$

With this model for $\Phi_{v v}$, the final formulation of Lysak's spectrum of the TWP reads :

$$
\begin{aligned}
\Phi_{p p}(k, \omega) & =(2 \pi)^{3} 3 \rho^{2} \int_{0}^{\infty}\left(\frac{\mathrm{d} U}{\mathrm{~d} y}\right)^{2} \frac{\left\langle v^{2}\right\rangle}{k_{e}^{3}}\left(\frac{k_{x}}{k_{e}}\right)^{2}\left[1+|k|^{2} / k_{e}^{2}\right]^{-17 / 6} \exp (-2|k| y) \delta\left(\omega+U(y) k_{x}\right) \mathrm{d} y \\
\phi_{p p}(\omega) & =2 \pi 3 \rho^{2} \int_{0}^{\infty}\left(\frac{\mathrm{d} U}{\mathrm{~d} y}\right)^{2}\left\langle v^{2}\right\rangle \omega^{2} U^{-3} k_{e}^{-4} I\left(\frac{\omega}{U k_{e}}, k_{e} y\right) \mathrm{d} y \\
I(\xi, \alpha) & =\int_{\mathbb{R}} \exp \left(-2 \alpha \sqrt{\xi^{2}+\zeta^{2}}\right) \times\left(1+\xi^{2}+\zeta^{2}\right)^{-17 / 6} \mathrm{~d} \zeta
\end{aligned}
$$

Lysak's original model is restricted to the very specific case of round pipe flows for which an analytic solution of the profiles of $U,\left\langle v^{2}\right\rangle$ and $k_{e}$ is known. An extension to an arbitrary boundary layer computed with a RANS solver was developed by Aupoix[31], deducing $k_{e}$ and $\left\langle v^{2}\right\rangle$ from the mean turbulent quantities :

$$
\begin{aligned}
k_{e} & =1.8 \varepsilon k_{t}^{-3 / 2} \\
\left\langle v^{2}\right\rangle & =-1.3\langle u v\rangle
\end{aligned}
$$

with $k_{t}$ the turbulent kinetic energy and $\varepsilon$ the dissipation rate. For the remainder of the paper, this model will be denoted as the LA model.

\subsection{Modification of the frequency part of the velocity spectrum}

Considering $\Phi_{p p}(k, \omega)$ given in eq. (5) only allows to compute the frequency dependency of the wall pressure spectrum $\phi_{p p}(\omega)$. As mentioned earlier, with this approach the spatial dependency has to be known to compute the radiated noise. This drawback was identified by Lysak where it was shown that the longitudinal correlation length provided by its model is zero, which is physically meaningless. This behaviour is caused by the Dirac distribution used to represent the frequency part of the velocity spectrum and that relies on Taylor's frozen turbulence hypothesis. For a given frequency and a given height in the boundary layer, only the advective wave number $-\omega / U$ is taken into account. This selectivity is due to the zero width of the Dirac distribution. To solve this issue, a wider distribution may be used. Cauchy distributions corresponding to an exponential decay in time associated to a width, set through a characteristic time of decorrelation caused by turbulence, are retained to this end.

Such an exponential decay in time was already used by Tam[41] in order to represent the decorrelation of the velocity fluctuation in jet flows. It was also used by Ewert et al.[42-44] to mimic the evolution of the velocity fluctuations in a boundary layer. In both works, the characteristic time of decorrelation $\tau_{S}$ is computed from RANS values. In particular Ewert et al. use :

$$
\tau_{S}=4.2 / w_{t}
$$

Where $w_{t}$ is the specific dissipation rate. This formulation will be used thereafter.

Finally, the Cauchy distribution in $k$ centred around the advective wave-number and having a width of $1 / U \tau_{s}$ reads:

$$
\Phi_{A}(k, y, \omega)=\frac{\tau_{s} / \pi}{1+\tau_{s}^{2}\left(\omega+U k_{x}\right)^{2}}
$$


The resulting $\Phi_{p p}$ model is reformulated as:

$$
\begin{aligned}
\Phi_{p p}(k, \omega) & =(2 \pi)^{3} 3 \rho^{2} \int_{0}^{\infty}\left(\frac{\mathrm{d} U}{\mathrm{~d} y}\right)^{2} \frac{\left\langle v^{2}\right\rangle}{k_{e}^{3}} \Phi_{T}(k, y) \Phi_{A}(k, y, \omega) \Phi_{D}(k, y) \mathrm{d} y \\
\Phi_{T}(k, y) & =\left(\frac{k_{x}}{k_{e}}\right)^{2}\left[1+|k|^{2} / k_{e}^{2}\right]^{-17 / 6} \\
\Phi_{D}(k, y) & =\exp (-2|k| y)
\end{aligned}
$$

For the remainder of the paper, this model will be denoted as the LMA model. This model is now analysed and departures from standard approaches such as Corcos model Eq.2 are evidenced to highlight the strengths and weaknesses of the models.

\subsection{Wave-number dependency}

For a given height $y$ in the boundary layer, function $k \rightarrow \Phi_{p p}[y](k, \omega)$ is proportional to the product $\Phi_{T} \Phi_{A} \Phi_{D}$. The three functions $\Phi_{T}(k, y), \Phi_{A}(k, y, \omega)$ and $\Phi_{D}(k, y)$ can be seen as filters in the wave-number domain. They will be referred to as "Turbulence filter", "Advection filter" and "Damping filter". The significant contributions to $\Phi_{p p}[y](k, \omega)$ in the $\left(k_{x}, k_{z}\right)$ plan result from a balance between these three filters. First, filters are analysed separately. Each of these filters is represented by its central wave-number $k_{0}$ and its $-3 \mathrm{~dB}$ wave-number bandwidth $\Delta k_{0}$.

\subsubsection{Turbulence filter}

The turbulence filter is a pass-band filter in the wave-number domain such that its central wave-number $k_{0} \propto k_{e}$. Figure 1 shows two representations of the turbulent filter through functions $k_{x} \rightarrow \Phi_{T}\left(k_{x}, 0\right)$ and $k_{x} \rightarrow \int \Phi_{T}\left(k_{x}, k_{z}\right) \mathrm{d} k_{z}$. Similar behaviours are observed with a slight shift of the central wave-number $k_{0}$. Their respective bandwidths $\Delta k_{0}$ are summarised in table 1.

\subsubsection{Advection filter}

The advection filter is also a pass-band filter. It only depends on $k_{x}$ and is a Cauchy distribution. It has a central wave-number $k_{0}=-\omega / U$ and has a bandwidth $\Delta k_{0}=2 \sqrt{\sqrt{2}-1} /\left(U \tau_{s}\right)$. Note that the bandwidth $\Delta k_{0}$ does not depend on the frequency $\omega$. A representation of the shape of the advection filter is given in figure 2 .

\subsubsection{Damping filter}

The damping filter is an exponential low-pass filter using the local height inside the boundary layer $y$ as a reference length.

\subsubsection{Resulting filter}

The wave-number selectivity of the LMA model depends on the competition between those three filters. The different regimes of $k \rightarrow \Phi_{p p}(k, \omega)$ when $\omega$ varies depend on the advection filter as it is the only one with a dependency toward $\omega$. At least three regimes can be found qualitatively. The advection filter has a narrower band-width than the turbulence filter. Thus there is a range of $\omega$ such as $\Phi_{p p}(k, \omega)$ is driven by the advection filter which corresponds to Corcos' description. For low frequencies, the advection filter is no longer a pass-band filter and becomes a low-pass filter. The turbulence filter is the only remaining band-pass filter and therefore $\Phi_{p p}(k, \omega)$ is therefore ruled by the turbulence filter. For high frequencies, $\omega / U \gg 1 / y$ and $\omega / U \gg k_{e}$ the advection filter will be damped by the turbulence and damping filters. A more detailed discussion on this competition is made in sub-section 2.5 .

The bandwidths of the three filters are compared in figure 3 versus the height of the TBL. The advection bandwidth is about one order of magnitude finer than the turbulence bandwidth. For a large of the TBL the advection bandwidth scales on the height of the TBL.

\subsection{Frequency analysis}

Aupoix[31] showed that the LA model is able to correctly reproduce the spectrum $\phi_{p p}(\omega)$ for a zero pressure gradient boundary layer. As the LMA model derives from the LA model, similar results are expected. Figures 4 and 5 show comparisons between several models for the configuration studied by Gravante[45]: a zero pressure gradient TBL flow over a flat plate with $U_{e}=15.3 \mathrm{~m} . \mathrm{s}^{-1}$ and $R_{\theta}=7076$. Goody[11] and Rozenberg[13] models are depicted on this figure to serve as references since they are standard correlations commonly used in the literature. For LA and LMA models, the spectra are computed as: 


$$
\phi_{p p}(\omega)=\frac{1}{(2 \pi)^{2}} \int \Phi_{p p}(k, \omega) \mathrm{d} k
$$

Figures 4 and 5 show no major difference between LA and LMA models in the range $\omega=\left[2.10^{3}, 2.10^{5}\right] \mathrm{s}^{-1}$. Rozenberg's model provides a similar trend for $\phi_{p p}(\omega)$. For high frequencies, all models exhibit different asymptotic behaviours. However, in the present study aimed at computing the radiated noise $I(\omega)$, the low amplitudes encountered at high frequencies will have a negligible contribution. In the present study, the observed differences are not relevant and will not be further discussed. For low frequencies a significant difference appears. LA, Goody and Rozenberg models show a $\omega^{2}$ trend, coherent with asymptotic behaviours as reminded in the introduction. The LMA model acts differently. For $\omega \rightarrow 0, \phi_{p p}(\omega)$ tends to a constant non-zero value. There is a priori no reason to have $\phi_{p p}(0)=0$, i.e. $\int S_{p p}(\tau) \mathrm{d} \tau=0$. The power spectral density function can be non-zero for $\omega=0$ : as long as the amplitude of the pressure fluctuations are zero, there will be no modification of the mean pressure value. Accurate measurements at such low frequencies are delicate. Few data are available in the literature. Analysis of TBL for large $R_{\theta}$ values by Tsuji et al. [46] exhibit non zero $\phi_{p p}(\omega)$ values for $\omega \rightarrow 0$, but no clear conclusion can be drawn so far on that specific point. One can note that if the advection filter bandwidth $\Delta k_{0}$ is reduced, the Cauchy distribution tends to a Dirac distribution and the $\omega^{2}$ behaviour of the LA model is recovered by the LMA model. The loss of the $\omega^{2}$ behaviour with non-frozen turbulence has been reported by Hu et al. [47]. The theoretical $\omega^{2}$ behaviour is established under the assumption of Taylor's hypothesis. The starting point is the Kraichnan-Phillips theorem

asserting $\Phi_{p p}(k, \omega) \underset{k \rightarrow 0}{\sim} k^{2}$. Then using Taylor's hypothesis and integrating over $k$, it can then be shown that $\phi_{p p}(\omega) \underset{\omega \rightarrow 0}{\sim} \omega^{2}$ See Howe[48, 49] for more details. As this paper does not use Taylor's hypothesis, this result can not be used anymore

LMA and LA models behave differently for high frequencies and $\tau_{S}$ is the key parameter that drives this modification. $\tau_{S}$ is defined by $A / \omega_{t}$ with $A=4.2$. Tests have been performed for $A \in[42 ; 0.42]$. The resulting compensated spectra $\left(\omega \phi_{p p}(\omega)\right)$ are presented in figure 6 . For $A<4.2$, the compensated spectrum shape is flattened and is shifted toward high frequencies. For $A>4.2$, the LMA model tend to the LA model. It has been shown that despite this change of shape the rms value $(2 \pi)^{-1} \int \phi_{p p}(\omega) \mathrm{d} \omega$ is almost independent of $A$ as its relative variation is below $1 \%$.

\subsection{Three branches diagram}

After briefly showing the capability of the LMA model to compute $\phi_{p p}(\omega)$, the focus is now made on the full spectrum $\Phi_{p p}(k, \omega)$ given by this model.

In standard approaches, as Corcos's model is only based on an advection mechanism, it is logical that for a given frequency $\omega$, function $k \rightarrow \Phi_{p p}(k, \omega)$ has its maximum at $k=\left(-k_{c}, 0,0\right)$. With the introduction of new characteristic lengths in the LMA model, the maxima of the longitudinal spectrum $k_{x} \rightarrow \int \Phi_{p p}(k, \omega) \mathrm{d} k_{z}$ will no longer depend solely on $k_{c}$. Figure 7 is a schematic representation of the location of the maxima for a TBL evolving along a flat plate without any pressure gradient. This figure was built observing spectra $\Phi_{p p}(k, \omega)$ of about a thousand zero pressure gradient TBL with $U_{e} \in[20 ; 50] \mathrm{m}^{-1}{ }^{-1}$ and $R_{\theta} \in\left[2.10^{3} ; 4.10^{4}\right]$ using the LMA model. A representation using a sketch was preferred since the axis can be scaled, allowing to highlight different trends on a single figure.

The fundamental difference with Corcos's model is that for a given frequency several maxima can be found. These maxima are located on three separate branches:

. $A F I_{2} H$ line in red which is denoted as the "advection branch",

- $E I_{1} C$ line in blue which is denoted as the "bifurcation branch",

. $B D$ line in green which is denoted as the "mirror branch".

For a given frequency $\omega$, the location of the maximum on each branch, if it exists, is denoted $k_{a}(\omega), k_{u}(\omega)$ and $k_{m}(\omega)$. These three branches can be described by nine characteristics points $A$ through $H$ and $I_{1}$ and $I_{2}$ and will be studied in details thereafter. Each of these points is fully characterised by a frequency and a wave-number.

Two lines have been added for reference:

- The advection line in purple such that $k+k_{c}=0$,

- The sonic line in magenta such that $k+k_{0}=0$.

\subsubsection{Advection branch}

The advection branch is similar to the one found using Corcos's model between points $F$ and $H$. The dispersion relation linking $\omega$ and $k_{x}$ is $\omega=A k_{a}(\omega)$. For Corcos's model, $A=-U_{c}$. It can be shown that the slope found using the LMA model is very close to $U_{c}=0.7 U_{e}$. However the advection branch looks different for small frequencies as it does not reach the origin since $k_{a}(0) \neq 0$, as represented by point $A$ in figure 7 . This behaviour is more physical than a collapse to the origin. 
Such a situation shows that enormous structures, larger than the boundary layer thickness, would be the most representative. The advection part and the small frequencies part of the advection branch can be separated easily because there is always a frequency $\omega$ such as $\mathrm{d} k_{a} / \mathrm{d} \omega(\omega)=0$ which is represented by point $F$ in figure 7 .

The advection branch also behaves differently at high frequencies. For Corcos's model, the maximum is always held at the line $-\omega / U_{c}$. Once again, this means that very small structures, possibly smaller than Kolmogorov's scale, are carrying the maximum of energy. For the present model, the advection branch ceases to exist above a given threshold frequency which is represented by point $H$ in figure 7 .

\subsubsection{Bifurcation branch}

Above a given frequency $\omega_{E}$ between $\omega_{F}$ and $\omega_{H}, k_{x} \rightarrow \int \Phi_{p p}(k, \omega) \mathrm{d} k_{z}$ has two maxima for $k_{x}<0$. This situation corresponds to the emergence of the bifurcation branch and is represented by point $E$ in figure 7. As above $\omega_{H}$, the advection branch ceases to exist, it means that there is a frequency $\omega_{I}$ such that $\int \Phi_{p p}\left(k_{a}\left(\omega_{I}\right), k_{z}, \omega_{I}\right) \mathrm{d} k_{z}=\int \Phi_{p p}\left(k_{u}\left(\omega_{I}\right), k_{z}, \omega_{I}\right) \mathrm{d} k_{z}$ and beyond $\omega_{I}$, the bifurcation branch dominates the advection branch. Finally, $k_{u}(\omega)$ tends to a finite value $k_{C}$ for $\omega \rightarrow \infty$. $k_{C} \neq 0$ for the same reason that $k_{A} \neq 0$.

\subsubsection{Mirror branch}

The advection and bifurcation branches are located on the left part of the diagram, i.e. for $k_{x}<0$, in figure 7. For all frequencies, a maximum can be found in the right part for $k_{x}>0$. It goes from the finite value $k_{B}$ for $\omega=0$ to the finite value $k_{D}$ for $\omega \rightarrow \infty$. It is found that $k_{A}+k_{B}=k_{C}+k_{D}=0$ and therefore the mirror branch behaves similar to the advection branch for low frequencies and the bifurcation branch for high frequencies.

\subsubsection{Sonic line}

The sonic line stands as a limit of validation for the LMA model since the incompressibility hypothesis was used to model the turbulent fluctuations. Consequently, the locations of the sonic line and the different branches must be compared before analysing results. At a given $k_{x}$ position, the advection line is always located below the sonic line for subsonic configurations. However the section of the bifurcation line above the sonic line must be ignored since it relies on an inconsistent hypothesis. The limiting case for which the bifurcation branch must not be considered entirely corresponds to point $E$, located on the sonic line.

\subsubsection{Scaled correlations}

From a series of zero pressure gradient TBL computations, the localisation of points $A, B, C, D, E, F, I_{1}$ and $I_{2}$ can be expressed using $R_{\theta}$. The wave-numbers are made dimensionless by using Reynolds numbers such as $R_{1 / k}=\frac{U}{k v}$ and $R_{U / \omega}=\frac{U^{2}}{\omega v}$ for frequencies. The correlations are summed up in table 2 .

The most interesting fact about these correlations concerns points $F$ and $E$. They can be seen as bounds for the validity of Corcos's model as according to the LMA model, the advection branch match the advection line for frequencies $\omega_{F}<\omega<\omega_{E}$. These correlations also provide the location of point $E$ with respect to the sonic line. The limit case for which the bifurcation branch must not be accounted for is reached when $\omega_{E}=c k_{E}$ i.e. $R_{U / \omega}=M R_{1 / k}$ or $M=0.7191 R_{\theta}^{-0.057}$.

\section{VIBRO-ACOUSTICS MODELLING}

The radiated noise is characterised through its acoustic intensity $p v$ integrated over all the vibrating surface. In order to have a frequency description of this quantity, the Fourier transform of the cross-correlation between the acoustic pressure and the acoustic velocity is considered:

$$
I(\omega)=\int_{\Omega} \mathcal{F}_{\tau}\left(\mathbb{E}_{t}\left[p_{a}(r, t) \times v_{a}(r, t+\tau)\right]\right) \mathrm{d} r
$$

The computational process is as follows. The acoustic quantities $p_{a}$ and $v_{a}$ are described using Green's functions and the fluctuating pressure $p$ as a source term. Therefore $I(\omega)$ Eq.(11) is expressed using the pressure spectrum $\Phi_{p p}$ and the Green's functions. In the present approach, the Green's functions are computed using the harmonic framework classically used in vibro-acoustics. Only two stochastic quantities will be manipulated : $I$ and $\Phi_{p p}$ whereas it is common in the literature to use intermediaries such as $\Phi_{w w}, \Phi_{v_{a} v_{a}}$ or $\Phi_{p_{a} p_{a}}$.

The final formulation of $I(\omega)$ cannot be used directly to get a numerical evaluation because the associated CPU time would be too large. A computational strategy is developed in order to get an accurate approximation of $I(\omega)$ with a computational cost compatible with industrial constraints. All these steps are described hereinafter. 


\subsection{Turbulence and Vibro-acoustic Coupling}

As explained before, $p_{a}$ and $v_{a}$ cannot be modelled as harmonic oscillating quantities because they are created by turbulence and can therefore only be described from a stochastic point of view. The modelling of $I(\omega)$ is divided into two steps. First, the stochastic part of Eq.(11) is extracted and isolated. Second, the remaining is computed using the harmonic oscillating

framework. This splitting is realized using Green's functions $g_{p}$ and $g_{v}$. As $p_{a}$ and $v_{a}$ are caused by the TWP, and assuming homogeneity in time, they are written as:

$$
\begin{aligned}
& p_{a}(r, t)=\iint p\left(r-x_{1}, t-\tau_{1}\right) g_{p}\left(r, r-x_{1}, \tau_{1}\right) \mathrm{d} x_{1} \mathrm{~d} \tau_{1} \\
& v_{a}(r, t)=\iint p\left(r-x_{2}, t-\tau_{2}\right) g_{v}\left(r, r-x_{2}, \tau_{2}\right) \mathrm{d} x_{2} \mathrm{~d} \tau_{2}
\end{aligned}
$$

Replacing these expressions in Eq.(11) and using the commutativity of the integrations with the expectation operator, $I(\omega)$ reads:

$$
I(\omega)=\iiint \int_{\Omega} \mathcal{F}_{\tau}\left(\mathbb{E}_{t}\left[p\left(r-x_{1}, t-\tau_{1}\right) p\left(r-x_{2}, t+\tau-\tau_{2}\right)\right]\right) g_{p}\left(r, r-x_{1}, \tau_{1}\right) g_{v}\left(r, r-x_{2}, \tau_{2}\right) \mathrm{d} r \mathrm{~d} x_{1} \mathrm{~d} \tau_{1} \mathrm{~d} x_{2} \mathrm{~d} \tau_{2}
$$

Using the notation

$$
\mathbb{E}_{t}[p(r, t) p(r+x, t+\tau)]=S_{p p}[r](x, \tau)
$$

Eq.(13) reads

$$
\begin{aligned}
I(\omega) & =\iiint \iint_{\Omega} \mathcal{F}_{\tau}\left(S_{p p}\left[r-x_{1}\right]\left(x_{1}-x_{2}, \tau+\tau_{1}-\tau_{2}\right)\right. \\
& \left.\times\left[g_{p}\left(r, r-x_{1}, \tau_{1}\right) g_{v}\left(r, r-x_{2}, \tau_{2}\right)\right]\right) \times \mathrm{d} r \mathrm{~d} x_{1} \mathrm{~d} \tau_{1} \mathrm{~d} x_{2} \mathrm{~d} \tau_{2}
\end{aligned}
$$

The Fourier transform relative to $\tau$ is computed, changing the integrations over $\tau_{1}$ and $\tau_{2}$ in Fourier transforms:

$$
I(\omega)=\iiint_{\Omega} \Phi_{p p}\left[r-x_{1}\right]\left(x_{1}-x_{2}, \omega\right) g_{p}\left(r, r-x_{1},-\omega\right) g_{v}\left(r, r-x_{2}, \omega\right) \mathrm{d} r \mathrm{~d} x_{1} \mathrm{~d} x_{2}
$$

with $g_{p}\left(r, r-x_{1}, \omega\right)=\mathcal{F}_{\tau}\left[g_{p}\left(r, r-x_{1}, \tau\right)\right]$ and $g_{v}\left(r, r-x_{2}, \omega\right)=\mathcal{F}_{\tau}\left[g_{v}\left(r, r-x_{2}, \tau\right)\right]$. Then $\Phi_{p p}$ is expressed as an inverse Fourier Transform in $x_{2}$ :

$$
\begin{aligned}
\Phi_{p p}[r](x, \omega) & =\int \Phi_{p p}[r](k, \omega) \frac{\exp (j k x)}{(2 \pi)^{2}} \mathrm{~d} k \\
I(\omega) & =\iiint_{\Omega} \int \Phi_{p p}\left[r-x_{1}\right](k, \omega) \frac{\exp \left(j k\left(x_{1}-x_{2}\right)\right)}{(2 \pi)^{2}} \mathrm{~d} k g_{p}\left(r, r-x_{1},-\omega\right) g_{v}\left(r, r-x_{2}, \omega\right) \mathrm{d} r \mathrm{~d} x_{1} \mathrm{~d} x_{2}
\end{aligned}
$$

Spatial homogeneity $\Phi_{p p}\left[r-x_{1}\right](k, \omega)=\Phi_{p p}(k, \omega)$ is assumed meaning that the boundary layer is evolving slowly in the longitudinal direction. Therefore, spatial Fourier transforms with respect to $x_{1}$ and $x_{2}$ can be computed as:

$$
I(\omega)=\iint_{\Omega} \Phi_{p p}(k, \omega) \frac{1}{(2 \pi)^{2}} g_{p}(r, k,-\omega) g_{v}(r,-k, \omega) \mathrm{d} r \mathrm{~d} k
$$

with $g_{p}(r, k, \omega)=\mathcal{F}_{x}\left[g_{p}\left(r, x_{1}, \omega\right)\right]$ and $g_{v}(r, k, \omega)=\mathcal{F}_{x}\left[g_{v}\left(r, x_{2}, \omega\right)\right]$. Equation(18) is the product of three terms: 
- $\Phi_{p p}[r](k, \omega)$, the TWP spectrum computed in section 2 ,

- $g_{p}(r, k,-\omega)$, the Green's function between TWP and the acoustic pressure,

- $g_{v}(r,-k, \omega)$, the Green's function between TWP and the acoustic velocity.

The determination of these Green's functions is done in the harmonic oscillating framework as is usual in vibro-acoustics.

\subsection{Vibro-acoustics Tools}

The determination of the Green's functions $g_{p}$ and $g_{v}$ may be decomposed in several steps assuming a unitary exciting TWP. First, $p_{a}$ is related to $v_{a}$ through the resolution of Helmholtz's equation also known as Rayleigh's integral:

$$
p_{a}(r, \omega)=\frac{\rho j \omega}{(2 \pi)^{2}} \int \frac{v_{a}(k, \omega) \exp (j r k)}{\sqrt{\left(\frac{\omega}{c}\right)^{2}-k^{2}}} \mathrm{~d} k
$$

The existence of a modal in vaccuo decomposition of the displacement $w$ is assumed. As the vibrating structure is a bidimensional plate, the modal decomposition is indexed by a couple of integers $(m, n)$. This decomposition is assumed to be orthonormal for the scalar product $\langle f, g\rangle=\int_{\Omega} f(r) g(r) \mathrm{d} r$. The natural frequency of the mode $(m, n)$ is denoted $\omega_{m n}$. Using this modal formalism, $p_{a}$ and $v_{a}$ read:

$$
\begin{aligned}
v_{a}(r, t) & =\frac{\partial w}{\partial t}(r, t) \\
w(r, \omega) & =\sum_{m n} a_{m n}(\omega) w_{m n}(r) \\
v_{a}(r, \omega) & =\sum_{m n} j \omega a_{m n}(\omega) w_{m n}(r) \\
p_{a}(r, \omega) & =\sum_{m n} b_{m n}(\omega) w_{m n}(r)
\end{aligned}
$$

The dynamical equilibrium in $r$ of the plate excited in $r^{\prime}$ reads:

$$
\text { Elasticity }+ \text { Damping }+ \text { Inertia }=\text { Turbulent Excitation }+ \text { Acoustical retroaction }
$$

which can be expressed using the modal formalism as:

$$
\sum_{m n} a_{m n}(\omega) \rho_{p} h\left[\omega_{m n}^{2}(1+j \eta)-\omega^{2}\right] w_{m n}(r)=\delta\left(r-r^{\prime}\right)+2 \sum_{m n} b_{m n}(\omega) w_{m n}(r)
$$

where $\eta$ is a damping loss factor, $\rho_{p}$ is the density of the plate and $h$ is the thickness of the plate.

Eq.(19) and Eq.(22) are projected on a given mode $w_{r s}$ and integrated over $\Omega$. Using the orthogonality property of the decomposition basis $\int_{\Omega} w_{m n}(r) w_{r s}(r) \mathrm{d} r=\delta_{m r} \delta_{n s}$, and the uncoupling modes approximation, meaning that the acoustical retroaction of the mode $(r, s)$ only impacts itself, Eq.(19) and Eq.(22) reduce to:

$$
\begin{gathered}
b_{r s}(\omega)=\frac{j \rho \omega^{2}}{(2 \pi)^{2}} \sum_{m n} a_{m n}(\omega) \iint \frac{w_{m n}(k) \exp (j k r) w_{r s}(r)}{\sqrt{\left(\frac{\omega}{c}\right)^{2}-k^{2}}} \mathrm{~d} k \mathrm{~d} r \\
=\frac{j \rho \omega^{2}}{(2 \pi)^{2}} \sum_{m n} a_{m n}(\omega) \int \frac{w_{m n}(k) w_{r s}(-k)}{\sqrt{\left(\frac{\omega}{c}\right)^{2}-k^{2}}} \mathrm{~d} k \\
a_{r s}(\omega) \rho h\left[\omega_{r s}^{2}(1+j \eta)-\omega^{2}\right]=w_{r s}\left(r^{\prime}\right)+2 b_{r s}(\omega)
\end{gathered}
$$

The summation in Eq.(23) vanishes and the equation ruling $a_{r s}$ then reads: 


$$
\begin{aligned}
a_{r s}(\omega) \rho h\left[\omega_{r s}^{2}(1+j \eta)-\omega^{2}\right] & =w_{r s}\left(r^{\prime}\right)+2 \frac{j \rho \omega^{2}}{(2 \pi)^{2}} a_{r s}(\omega) \int \frac{w_{r s}(k) w_{r s}(-k)}{\sqrt{\left(\frac{\omega}{c}\right)^{2}-k^{2}}} \mathrm{~d} k \\
& =w_{r s}\left(r^{\prime}\right)+2 j \omega a_{r s}(\omega) Z_{r s}(\omega) \\
\Leftrightarrow a_{r s}(\omega) \mathcal{A}_{r s}(\omega) & =w_{r s}\left(r^{\prime}\right)
\end{aligned}
$$

with $Z_{r s}(\omega)=\frac{\rho \omega}{(2 \pi)^{2}} \int\left|w_{r s}(k)\right|^{2}\left[\left(\frac{\omega}{c}\right)^{2}-k^{2}\right]^{-1 / 2} \mathrm{~d} k$ the radiation impedance and $\mathcal{A}_{r s}(\omega)=\rho h\left[\omega_{r s}^{2}(1+j \eta)-\omega^{2}\right]-2 j \omega Z_{r s}(\omega)$ the structural impedance.

Finally, the Green's functions are computed from $a_{r s}$ :

$$
\begin{aligned}
& g_{p}\left(r, r^{\prime}, \omega\right)=\sum_{m n} b_{m n}(\omega) w_{m n}(r)=\sum_{m n} j \omega w_{m n}\left(r^{\prime}\right)\left[1 / \mathcal{A}_{m n}(\omega)\right] Z_{m n}(\omega) w_{m n}(r) \\
& g_{v}\left(r, r^{\prime}, \omega\right)=j \omega \sum_{m n} a_{m n}(\omega) w_{m n}(r)=j \omega \sum_{m n} w_{m n}\left(r^{\prime}\right)\left[1 / \mathcal{A}_{m n}(\omega)\right] w_{m n}(r)
\end{aligned}
$$

Applying a Fourier transform towards $r^{\prime}$, the Green's functions appearing in Eq.(18) are computed through:

$$
\begin{aligned}
g_{p}(r, k,-\omega) & =\sum_{m n}-j \omega w_{m n}(k)\left[1 / \mathcal{A}_{m n}(\omega)\right] Z_{m n}(-\omega) w_{m n}(r) \\
g_{v}(r,-k, \omega) & =j \omega \sum_{m n} w_{m n}(-k)\left[1 / \mathcal{A}_{m n}(\omega)\right] w_{m n}(r)
\end{aligned}
$$

Since $\mathcal{A}_{m n}(\omega)$ and $w_{m n}(k)$ are Fourier transforms of real quantities, we have $\mathcal{A}_{m n}(\omega) \mathcal{A}_{m n}(-\omega)=\left|\mathcal{A}_{m n}(\omega)\right|^{2}$ and $w_{m n}(k) w_{m n}(-k)=$ $\left|w_{m n}(k)\right|^{2}$. Using the orthogonality of the modal decomposition, the acoustic intensity finally reads:

$$
\begin{aligned}
I(\omega) & =\frac{\omega^{2}}{(2 \pi)^{2}} \sum_{m n}\left[\int \Phi_{p p}(k, \omega)\left|w_{m n}(k)\right|^{2} \mathrm{~d} k\right] \frac{Z_{m n}(-\omega)}{\left|\mathcal{A}_{m n}(\omega)\right|^{2}} \\
& =\frac{\omega^{2}}{(2 \pi)^{2}} \sum_{m n}\left[K_{m n}(\omega)\right] Z_{m n}(-\omega)\left|\mathcal{A}_{m n}(\omega)\right|^{-2}
\end{aligned}
$$

\subsection{Computational Strategy}

The numerical evaluation of the radiated noise from Eq. (27) is practically unachievable due to the large number of operations required for computation. Three terms are to be computed: $\mathcal{A}_{m n}, Z_{m n}$ and $K_{m n}$. The first two depend only on the mechanical characteristic of the exited structure and can be computed for a small computational cost and tabulated for later use. However, $K_{m n}$ depends on both flow characteristics and the geometry of the structure, requiring updates as flow conditions change. The evaluation of this term is the reason why $I(\omega)$ cannot be directly computed from Eq. (27). To justify this statement quantitatively, let's consider the special case of a rectangular plate $L_{x} \times L_{z}$ simply supported on its edges. The displacement modes are :

$$
\begin{aligned}
w_{m n}(r) & =w_{m}\left(r_{x}\right) w_{n}\left(r_{z}\right) \\
w_{m}\left(r_{x}\right) & =\sqrt{\frac{L_{x}}{2}} \sin \left(m \pi \frac{r_{x}}{L_{x}}\right)=\sqrt{\frac{L_{x}}{2}} \sin \left(k_{m} r_{x}\right) \\
w_{m n}(k) & =w_{m}\left(k_{x}\right) w_{n}\left(k_{z}\right) \\
w_{m}\left(k_{x}\right) & =\frac{m \pi}{L_{x}} \sqrt{2 / L_{x}} \frac{\left((-1)^{m} e^{-j L_{x} k_{x}}-1\right)}{\left(k_{x}^{2}-m^{2} \pi^{2} L_{x}^{-2}\right)} \\
& =k_{m} \sqrt{2 / L_{x}} \frac{\left((-1)^{n} e^{-j L_{x} k_{x}}-1\right)}{\left(k_{x}^{2}-k_{m}^{2}\right)}
\end{aligned}
$$


Eq.(28) and figure 8 indicate that $w_{m}\left(k_{x}\right)$ and consequently $\left|w_{m n}(k)\right|^{2}$ are oscillating functions. Therefore the computation of $K_{m n}$ which is an integration over the unbounded domain $\mathbb{R}^{2}$ reveals itself to be delicate. It is easier to perform the integration over each lobe of $\left|w_{m n}(k)\right|^{2}$ and to sum all the contributions. The domain $\mathbb{R}^{2}$ is meshed with rectangles. Each rectangle corresponds to one lobe of $\left|w_{m n}(k)\right|^{2}$ and is indexed by the two integers $M$ and $N$. The boundaries of the lobes are defined by :

$$
\begin{aligned}
& (-1)^{n} e^{-j L_{x} k_{x}}-1=0 \\
& (-1)^{m} e^{-j L_{z} k_{z}}-1=0
\end{aligned}
$$

and can easily be found depending on the parity of $m$ and $n$.

Numerical integrations on each lobe are computed using Shampine[50] adaptive quadrature which is able to compute indefinite integrals on unbounded and bidimensional supports. In reality, only a finite number of lobes is considered. The contributions of high values of $M$ or $N$ are negligible. Two bounded values $M_{L}$ and $N_{L}$ can be defined such that only lobes satisfying $M<M_{L}$ and $N<N_{L}$ are computed. $M_{L}$ and $N_{L}$ are found by comparing the wavelengths of the lobe $M \pi / L_{x}$ and $N \pi / L_{z}$ and a characteristic wavelength of the TWP $\max \left(0.7 \omega / U_{e}, k_{e}(y=\delta / 2)\right)$. It has been shown that by taking $M_{L}=N_{L}=100$, the relative error resulting from the computation of $K_{m n}$ is below $1.10^{-6}$.

From these considerations, a naive way to compute $I(\omega)$ can be elaborate using four loops. The associated computational process is summarized in algorithm 1.

Table 3 gives typical orders of magnitudes of the number of operations for each loop. The total number of operations for the naive algorithm is about $3.10^{14}$. In an industrial context, it is obviously impossible to rely on such a process to compute $I(\omega)$. A good approximation of $I(\omega)$ is however achievable, with a reasonable computational cost, because it can be shown that most of the contributions involved in the computation of terms $I_{1}, I_{2}$ and $I_{3}$ in algorithm 1 are negligible.

Table 3 also provides the number of operations required if only the major contributions are computed with a relative error on $I(\omega)$ below $10^{-3}$. The number of operations has been drastically reduced since only $10^{10}$ are required. The main difficulty is that the location in the different space domains of these major contributions is a priori unknown. An exploratory process is used to locate these contributions. For three of the loops involved in algorithm 1, the number of operations is reduced.

\subsubsection{Boundary layer integration loop}

The first loop is the integration along the boundary layer thickness. According to Eq. (9) $\Phi_{p p}(y, k, \omega)$ is proportional to $\left(\frac{\mathrm{d} U}{\mathrm{~d} y}\right)^{2} \frac{\left\langle v^{2}\right\rangle}{k_{e}^{3}}$. As $\left\langle v^{2}\right\rangle(y=0)=\left\langle v^{2}\right\rangle(y=\delta)=0$, the contributions of the near wall and frontier regions of the boundary layer can be neglected. The maximum of the function $y \rightarrow\left(\frac{\mathrm{d} U}{\mathrm{~d} y}\right)^{2} \frac{\left\langle v^{2}\right\rangle}{k_{e}^{3}}$ is roughly located near $\delta / 2$ and the function is monotonically decreasing in both directions. The idea is to begin the exploration at $y=\delta / 2$ and progress towards the wall until $I_{1}$ does not contribute significantly to $I_{2}$ and to repeat the process towards the frontier of the boundary layer. With a relative threshold fixed at $\varepsilon_{Y}=I_{1} / I_{2}=1.10^{-5}$ the number of heights to be considered in the boundary layer is reduced by a factor of 3 in subsonic boundary layer configurations with unitary Reynolds number in $\left[10^{6}, 10^{7}\right]$ which is characteristic of flows around helicopters.

\subsubsection{Wave number integration loop}

The second loop is the summation over $M, N$. It is quite difficult to know which of the $I_{2}$ terms contribute most to $I_{3}$. One can presume that $I_{2}(M, N, m, n, \omega)$ for $(M, N)=(m, n)$ has a significant contribution. However it can be shown that $(M, N)=(m, n)$ is not always the global maximum as other local maxima can exist. The search of all these maxima may be a complex process and is very time-consuming.

The idea followed in this study is to compute $I_{2}(M, N, m, n, \omega)$ for all couples $(\mathrm{M}, \mathrm{N}) \in\left[1: M_{L}\right] \times\left[1: N_{L}\right]$ with a less accurate but cheaper method of integration. This method does not need to be precise as its purpose is only to find the couples $(M, N)$ giving a significant $I_{2}$. The $I_{1}$ integrals are evaluated using a Gauss quadrature using $\left|w_{m n}(k)\right|^{2}$ as the weight function. Only $\Phi_{p p}(k, \omega)$ needs to be evaluated. The number of evaluations depends on the order of the quadrature. It has been shown that the zero-order, which requires only one evaluation per lobe, is sufficient to sort the $(M, N)$ couples and find the major contributors to $I_{2}(M, N, m, n, \omega)$. Once sorted, the main contributions are accurately computed using Shampine's quadrature. With a relative threshold fixed at $\varepsilon_{M N}=I_{2} / I_{3}=1 \cdot 10^{-5}$ only one percent of the initial amount of operations is required.

\subsubsection{Modal loop}

The last loop is the modal summation over $(m, n)$. From a vibro-acoustic point of view, two families of modes need to be considered: the resonant and the radiative modes. For a given frequency $\omega$, the radiative modes are such that there is a good 
coupling between the acoustic pressure and velocity which is obtained when $Z_{m n}(\omega)$ is maximised. The resonant modes have their natural frequency satisfying $\omega_{m n}=\omega$ and therefore $\left|\mathcal{A}_{m n}(\omega)\right|^{-2}$ will be maximal. The modes of these two families are expected to be significant contributors to $I(\omega)$ and the others significant contributors are supposed to be located in their neighbourhoods in the $(m, n)$ plan.

In the special case of a rectangular plate $L_{x} \times L_{z}$ simply supported on its edges, these families are localised on ellipses $\left(m \pi / L_{x}\right)^{2}+\left(n \pi / L_{z}\right)^{2}=C^{2}$. For resonant modes $C=k_{m n} \sqrt{\omega / \omega_{m n}}$ and for radiation modes $C=\omega / c$. There exists a frequency $\omega$ such that these two ellipses are superposed and therefore resonant and radiative modes are identical. $I(\omega)$ is expected to achieve a particularly high value in such cases.

Noticing that $\Phi_{p p}(k, \omega)$ is maximised when $k_{z}=0$, the longitudinal modes located on lines $n= \pm 1$ are also expected to be significant contributors.

Due to these considerations, there is no need to compute all the contributions of all the $(m, n)$ modes, only the most significant ones must be considered. Once again, their locations are a priori unknown but they are hypothesized to be located near the two ellipses and the two lines described above. The strategy is as follows:

1. Compute the contributions of the radiative, resonant and longitudinal modes.

2. Sort these contributions.

3. Explore the neighbourhood of these modes in order to find new significant contributors.

The threshold is fixed at $\varepsilon_{m n}=I_{3} / I=1.10^{-5}$. This allows a reduction of the number of computations of a factor 100 .

Following the rules explained above, the light algorithm 2 can be written.

The associated computational cost depends on the threshold values $\varepsilon_{Y}, \varepsilon_{M N}, \varepsilon_{m n}, M_{L}$ and $N_{L}$. Since computing $I(\omega)$ through the naive algorithm is not possible, the exact error of the light algorithm is not directly accessible. Therefore the threshold values cannot be directly related to that error. Nevertheless, an estimate of the error may be obtained as follows. The three summations of the algorithm are expressed with unknown errors $e_{1}, e_{2}$ and $e_{3}$.

$$
I_{2}=\sum I_{1}+e_{1} ; I_{3}=\sum I_{2}+e_{2} ; I=\sum I_{3}+e_{3}
$$

Assuming the existence of constants $A_{1}, A_{2}$ and $A_{3}$ such that $e_{i}<\min \left(I_{i}\right) A_{i}$, the errors are expressed using the threshold values $e_{1}<\varepsilon_{Y} I_{2} A_{1}, e_{2}<\varepsilon_{M N} I_{3} A_{2}$ and $e_{3}<\varepsilon_{m n} I A_{3}$.

Thus Eq.(30) yields:

$$
\begin{aligned}
I_{2} & <\left(\sum I_{1}\right) /\left(1-\varepsilon_{Y} A_{1}\right) \\
I_{3} & <\left(\sum I_{2}\right) /\left(1-\varepsilon_{M N} A_{2}\right) \\
I & <\left(\sum I_{3}\right) /\left(1-\varepsilon_{m n} A_{3}\right)
\end{aligned}
$$

Finally an upper bound on the error on $I$ is expressed as:

$$
\begin{aligned}
I-\sum \sum \sum I_{1} & <\left[\left(1-\varepsilon_{Y} A_{1}\right)\left(1-\varepsilon_{M N} A_{2}\right)\left(1-\varepsilon_{m n} A_{3}\right)\right]^{-1}-1 \\
E_{M} & =\left[\left(1-\varepsilon_{Y} A_{1}\right)\left(1-\varepsilon_{M N} A_{2}\right)\left(1-\varepsilon_{m n} A_{3}\right)\right]^{-1}-1 \\
& \approx\left(1+\varepsilon_{Y} A_{1}\right)\left(1+\varepsilon_{M N} A_{2}\right)\left(1+\varepsilon_{m n} A_{3}\right)-1 \\
& \approx \varepsilon_{Y} A_{1}+\varepsilon_{M N} A_{2}+\varepsilon_{m n} A_{3}
\end{aligned}
$$

Considering a set of random values of $\omega, m, n, M$ and $N$, tests show that $\varepsilon_{Y}=1.10^{-5}$ implies $\varepsilon_{Y} A_{1}<1.10^{-3}$. Using this value and considering a set of random values for $\omega, m$ and $n$, it has been shown that $\varepsilon_{M N}=1.10^{-5}$ implies $\varepsilon_{M N} A_{2}<1.10^{-3}$. Similarly, considering a set of random values of $\omega$, tests conducted with $\varepsilon_{m n}=1.10^{-5}$ lead to $\varepsilon_{m n} A_{3}<1.10^{-3}$. As a consequence, using $\varepsilon_{Y}=\varepsilon_{M N}=\varepsilon_{m n}=1.10^{-5}$, the relative error for the computation of $I(\omega)$ is estimated at $10^{-3}$.

\subsection{Application}

The purpose of the application case is to demonstrate the importance of the difference in the longitudinal dependency between the Corcos and LMA models highlighted in section 2 in the prediction of the radiated noise. Two cases will be compared. Both of them consist in a $0,25 \mathrm{~m} \times 0,17 \mathrm{~m}$ aluminium plate, simply supported on its edges and exited by a turbulent flow characterised by $U_{e}=50 \mathrm{~ms}^{-1}$ and $R_{\theta}=3540$. The difference between Case 1 and Case 2 rely on the thickness of the plates as $h_{1}=0.2 \mathrm{~mm}$ and $h_{2}=5 \mathrm{~mm}$. Therefore, the modal function $w_{m n}(k)$ and the radiation impedances are the same for both cases while the natural frequencies of the modes and $\mathcal{A}_{m n}(\omega)$ are different. 
In figure 7, symbols $\Delta$ (case 1 ) and $\square$ (case 2) show where the two cases are located in the $\left(k_{x}, \omega\right)$ plan. As it is only a sketch, figure 9 presents the actual numerical values for cases 1 and two. In addition, figure 9 presents the advection branch of the flow and the natural frequencies of mode $(1,1)$ of cases 1 and 2 . Below $200 \mathrm{~Hz}$, the maxima location of $k_{x} \rightarrow \Phi_{p p}(k, \omega)$ tends to be near $-80 \mathrm{~m}^{-1}$ for the LMA model whereas it linearly decreases for Corcos's model. Taking a mode with a natural longitudinal wave-number below $80 \mathrm{~m}^{-1}$, two situations arise. First, if its natural frequency is below $200 \mathrm{~Hz}$ (or located below point $\mathrm{F}$ in figure 7), the radiated noise due to this mode predicted using the LMA model will be much smaller than the one predicted by Corcos's model. Second, if its natural frequency is above $200 \mathrm{~Hz}$ (or located above point $\mathrm{F}$ in figure 7) and below the very high frequencies range, the radiated noise predicted by both models will have the same order of magnitude because the maxima are located at the same places. The results will not be exactly the same because of differences in the spectral width in the longitudinal and the transversal directions.

If the mode $(1,1)$ is used, its natural longitudinal wave-number is $12.56 \mathrm{~m}^{-1}$ and the associated natural frequencies are respectively $25 \mathrm{~Hz}$ and $633 \mathrm{~Hz}$. The radiation frequency which correspond to the maximum of $Z_{11}$ is $1209 \mathrm{~Hz}$. The radiated noise due to the mode $(1,1)$ is plotted versus the frequency for both Corcos and LMA models on figure 10. For each case, the radiated noise is scaled by $\max \left[I_{\text {LMA }}(\omega)\right]$.

As predicted, the behaviour is completely different if the natural frequency is above or below $200 \mathrm{~Hz}$. For case 2 , near the natural frequency, both models give the same radiated noise. One may notice that small differences begin to appears for frequencies far from the natural frequency of the mode $(1,1)$. But for such frequencies, the radiated noise is negligible in comparison with that is found for the natural frequency. For case 1, the behaviour is different as the shape is quite similar but the maxima differ from almost two orders in magnitude. This demonstrates that using Corcos's model for all frequencies can lead to large differences in the prediction of the radiated noise.

What has been shown on figure 10 can be refined to find precisely the transition between both regimes. Considering a serie of plates with a variable thickness such as their natural frequencies lies in $[20 \mathrm{~Hz} ; 1 \mathrm{kHz}]$, for each of these, the radiated noise at the natural frequency of both models will compared. Results are depicted on figure 11 where the ratio of the radiated noise versus the natural frequency of the plates.

The plate with the natural frequency $f_{m n} \approx 200 \mathrm{~Hz}$ is drawn to separate both regimes. One can notice that the radiated noise is maximal at natural frequencies due to high vibration level and the radiation frequency is far above and therefore not involve here.

The ratio between both radiated noises reaches values up to 60 which corresponds to $\approx 18 \mathrm{~dB}$. This value is not negligible for acoustics and shows that improved models must be developed to overcome Corcos's model limitations outside of its range of utilisation.

\section{Conclusion}

An analytical model of the fluctuations of the turbulent wall pressure was developed in order to improve the prediction of noise radiated by a vibrating structure excited by a turbulent boundary layer. Most of the models available in the literature are based on empirical descriptions of the TWP. In the LMA model, the TWP fluctuations are computed from the modelled turbulent velocity fluctuations, leading to a more general model as the empiricism is not used directly on the final quantity of interest.

The TWP fluctuations are described in time and space through the LMA model. Both aspects are required for vibro-acoustics applications whereas many studies focus only on the temporal aspects. The TWP is characterized by its spectrum in the frequency/wave number domain which is more convenient for vibro-acoustics applications rather than the cross-correlation in space and time.

This new model has similarities with the empirical models available in the literature such as Corcos model, mostly when advection is the main mechanism involved. However, strong differences arise for high and low frequencies where the influence of turbulence scales cannot be neglected. One of the main interest of this finding is to provide frequencies boundaries for models based only on the advection mechanism which are commonly used without any boundaries. Outside of these boundaries, using Corcos or LMA model to compute the radiated noise will lead to completely different results as their behaviours in the frequency wave-number domain are different.

The LMA model was coupled with classic vibro-acoustics models in order to compute the radiated noise emitted by a structure excited by a turbulent flow. Considering the use of the final model in an industrial context, a simplified version was developed. The computational cost was significantly reduced while the resulting error remains small and controlled.

The predicted new features found for the TWP spectrum must be confirmed experimentally. As the rare existing experimental data available in the literature are not documented enough to be reproduced with the present approach, the next step would be to design a new experimental apparatus in order to validate the different steps of the modelling. In particular, the TWP and the turbulent velocity fluctuations spectra should be measured simultaneously in the frequency/wave-number domain allowing the verification of the validity of the proposed model. 


\section{Nomenclature}

$a_{m n}$ modal amplitude of displacement

$\mathcal{A}_{m n}$ structure impedance

$b_{m n}$ modal amplitude of pressure

$c \quad$ speed of sound

$\mathrm{d} p / \mathrm{d} x$ pressure gradient

$g_{p} \quad$ Green function between TWP and the acoustic pressure

$g_{v} \quad$ Green function between TWP and the acoustic velocity

$h$ thickness of the vibrating plate

I acoustical intensity

$I_{1}, I_{2}, I_{3}$ partial summation of the acoustical intensity

$j \quad \operatorname{Re}(j)=0, \operatorname{Im}(j)=1$

$k \quad$ wave-number

$k_{c}=\omega / U$ advection wave-number

$k_{e} \quad$ wave number carrying the maximum of energy

$k_{x} \quad$ longitudinal wave-number

$k_{y}$ normal wave-number

$k_{z} \quad$ transversal wave-number

$K_{m n}$ turbulence impedance

$L_{x} \quad$ length of the vibrating plate

$L_{z} \quad$ width of the vibrating plate

$m, n$ modal indexes

$M, N$ lobe indexes

$M_{L}, N_{L}$ boundaries of lobe indexes

$p \quad$ turbulent wall pressure

$p_{a}$ acoustical wall pressure

$S_{p p}$ pressure inter-correlation

$t, \tau$ time

$u_{i}=(u, v, w)$ fluctuating fluid velocity

$u_{\tau} \quad$ friction velocity

$U_{e}$ free-stream velocity

$U_{i}=(U, V, W)$ mean fluid velocity

$v_{a}$ velocity of the plate

$\left\langle v^{2}\right\rangle=\mathbb{E}\left(v^{2}\right)$

$w$ displacement of the plate

$w_{m n}(k)$ modal wave-number distribution

$x, r$ position

$x \quad$ longitudinal coordinate

$y$ normal coordinate

$z$ transversal coordinate

$Z_{m n}$ radiation impedance

$\alpha, \beta$ Corcos's constants

$\delta \quad$ boundary layer thickness

$\delta()$ Dirac distribution

$\varepsilon \quad$ turbulent dissipation

$\varepsilon_{m n}$ modal threshold

$\varepsilon_{M N}$ lobe threshold

$\varepsilon_{Y} \quad$ altitude threshold

$\theta$ momentum thickness

$\rho \quad$ fluid density

$\rho_{P} \quad$ density of the vibrating plate

$\tau_{S} \quad$ characteristic time of decorrelation

$\Phi_{A}$ advection filter

$\Phi_{D}$ damping filter

$\phi_{p p}$ pressure power spectral density

$\Phi_{T}$ turbulence filter

$\omega$ pulsation

$\omega_{t}$ turbulent specific dissipation rate 
$\Omega \quad$ surface of the vibrating plate

$\mathcal{F}_{t}(f)=\int f(t) \exp (j \omega \tau) \mathrm{d} \tau$, Fourier Transform

$\mathcal{F}_{t}^{-1}(f)=(2 \pi)^{-1} \int f(t) \exp (-j \omega \tau) \mathrm{d} \tau$, inverse Fourier Transform

\section{References}

[1] Panton, R. L. and J. H. Linebarger (1974). Wall pressure spectra calculations for equilibrium boundary layers. Journal of Fluid Mechanics 65(2), 261-287.

[2] Blake, W. K. (1986). Mechanics of Flow-Induced Sound and Vibration: Complex Flow-Structure Interactions. Applied Mathematics and Mechanics Series. Academic Press.

[3] Bradshaw, P. (1967). Inactive motion and pressure fluctuations in turbulent boundary layers. Journal of Fluid Mechanics 30(2), 241-258.

[4] Keith, W. L., D. Hurdis, and B. Abraham (1992). A comparison of turbulent boundary layer wall-pressure spectra. Journal of Fluids Engineering 114(3), 338-347.

[5] Farabee, T. M. and M. J. Casarella (1991). Spectral features of wall pressure fluctuations beneath turbulent boundary layers. Physics of Fluids A: Fluid Dynamics (1989-1993) 3(10), 2410-2420.

[6] McGrath, B. E. and R. L. Simpson (1987). Some features of surface pressure fluctuations in turbulent boundary layers with zero and favorable pressure gradients.

[7] Blake, W. K. (1970). Turbulent boundary-layer wall-pressure fluctuations on smooth and rough walls. Journal of Fluid Mechanics 44(04), 637-660.

[8] Goody, M. C. and R. L. Simpson (2000). Surface pressure fluctuations beneath two-and three-dimensional turbulent boundary layers. AIAA journal 38(10), 1822-1831.

[9] Efimtsov, B. M. (1982). Characteristics of the field of turbulent wall pressure-fluctuations at large reynolds-numbers. SOVIET PHYSICS ACOUSTICS-USSR 28(4), 289-292.

[10] Efimtsov, B. M. (1984). Similarity criteria for the spectra of wall pressure fluctuations in a turbulent boundary layer. Akusticheskii Zhurnal 30, 58-61.

[11] Goody, M. (2004). Empirical spectral model of surface pressure fluctuations. AIAA journal 42(9), 1788-1794.

[12] Catlett, M., J. Forest, J. A. Anderson, and D. Stewart (2014). Empirical spectral model of surface pressure fluctuations beneath adverse pressure gradients. In Proceedings of the 20th AIAA/CEAS aeroacoustics conference.

[13] Rozenberg, Y., G. Robert, and S. Moreau (2012). Wall-pressure spectral model including the adverse pressure gradient effects. AIAA journal 50(10), 2168-2179.

[14] Hu, N. and M. Herr (2016). Characteristics of wall pressure fluctuations for a flat plate turbulent boundary layer with pressure gradients. In 22nd AIAA/CEAS Aeroacoustics Conference, pp. 2749.

[15] $\mathrm{Hu}$, N. (2017). Empirical spectral model of wall pressure fluctuations including adverse pressure gradient effects. In 23rd AIAA/CEAS Aeroacoustics Conference, pp. 3203.

[16] Kamruzzaman, M., D. Bekiropoulos, T. Lutz, W. Würz, and E. Krämer (2015). A semi-empirical surface pressure spectrum model for airfoil trailing-edge noise prediction. International Journal of Aeroacoustics 14(5-6), 833-882.

[17] Hwang, Y., W. K. Bonness, and S. A. Hambric (2009). Comparison of semi-empirical models for turbulent boundary layer wall pressure spectra. Journal of Sound and Vibration 319(1), 199-217.

[18] Lee, S. (2018). Empirical wall-pressure spectral modeling for zero and adverse pressure gradient flows. AIAA Journal, $1818-1829$.

[19] Corcos, G. M. (1963). Resolution of pressure in turbulence. The Journal of the Acoustical Society of America 35(2), 192-199.

[20] Corcos, G. M. (1964). The structure of the turbulent pressure field in boundary-layer flows. Journal of Fluid Mechanics 18(03), 353-378.

[21] Kraichnan, R. H. (1956). Pressure fluctuations in turbulent flow over a flat plate. The Journal of the Acoustical Society of America 28(3), 378-390.

[22] Lee, Y.-T., W. K. Blake, and T. M. Farabee (2005). Modeling of wall pressure fluctuations based on time mean flow field. Journal of Fluids Engineering 127(2), 233-240.

[23] Lee, Y.-T., T. M. Farabee, and W. K. Blake (2009). Turbulence effects of wall-pressure fluctuations for reattached flow. Computers \& Fluids 38(5), 1033-1041.

[24] Remmler, S., J. Christophe, J. Anthoine, and S. Moreau (2010). Computation of wall pressure spectra from steady flow data for noise prediction. AIAA journal 48(9), 1997-2007.

[25] Parchen, R. R. (1998). Progress report DRAW: A prediction scheme for trailing edge noise based on detailed boundary layer characteristics. TNO Institute of Applied Physics.

[26] Bertagnolio, F., H. Aagaard Madsen, and C. Bak (2009). Experimental validation of tno trailing edge noise model and application to airfoil optimization. In 2009 European Wind Energy Conference and Exhibition. 
[27] Bertagnolio, F., A. Fischer, and W. J. Zhu (2014). Tuning of turbulent boundary layer anisotropy for improved surface pressure and trailing-edge noise modeling. Journal of Sound and Vibration 333(3), 991-1010.

[28] Peltier, L. J. and S. A. Hambric (2007). Estimating turbulent-boundary-layer wall-pressure spectra from cfd rans solutions. Journal of Fluids and Structures 23(6), 920-937.

[29] Slama, M., C. Leblond, and P. Sagaut (2018). A kriging-based elliptic extended anisotropic model for the turbulent boundary layer wall pressure spectrum. Journal of Fluid Mechanics 840, 25-55.

[30] Lysak, P. D. (2006). Modeling the wall pressure spectrum in turbulent pipe flows. Journal of fluids engineering 128(2), 216-222.

[31] Aupoix, B. (2015). Extension of Lysak s Approach to Evaluate the Wall Pressure Spectrum for Boundary Layer Flows. Flow, Turbulence and Combustion 94(1), 63-78.

[32] Maury, C., P. Gardonio, and S. J. Elliott (2002). A wavenumber approach to modelling the response of a randomly excited panel, Part ii: Application to aircraft panels excited by a turbulent boundary layer. Journal of Sound and Vibration 252(1), 115-139.

[33] Graham, W. R. (1996a). Boundary layer induced noise in aircraft, Part i: The flat plate model. Journal of Sound and Vibration 192(1), 101-120.

[34] Graham, W. R. (1996b). Boundary layer induced noise in aircraft, Part ii: The trimmed flat plate model. Journal of Sound and Vibration 192(1), 121-138.

[35] Borisyuk, A. O. and V. T. Grinchenko (1997). Vibration and noise generation by elastic elements excited by a turbulent flow. Journal of Sound and Vibration 204(2), 213-237.

[36] Mazzoni, D. (2003). An efficient approximation for the vibro-acoustic response of a turbulent boundary layer excited panel. Journal of Sound and Vibration 264(4), 951-971.

[37] Davies, H. G. (1971). Sound from Turbulent-Boundary-Layer-Excited Panels. The Journal of the Acoustical Society of America 49(3B), 878-889.

[38] Hwang, Y. F. and G. Maidanik (1990). A wavenumber analysis of the coupling of a structural mode and flow turbulence. Journal of Sound and Vibration 142(1), 135-152.

[39] Maury, C., P. Gardonio, and S. J. Elliott (2002). A wavenumber approach to modelling the response of a randomly excited panel, part i: general theory. Journal of Sound and Vibration 252(1), 83-113.

[40] Lysak, P. D. and T. A. Brungart (2003). Velocity spectrum model for turbulence ingestion noise from computationalfluid-dynamics calculations. AIAA journal 41(9), 1827-1829.

[41] W. Tam, C. K. and L. Auriault (1999). Jet mixing noise from fine-scale turbulence. AIAA journal 37(2), 145-153.

[42] Ewert, R. (2008). Broadband slat noise prediction based on caa and stochastic sound sources from a fast random particle-mesh (rpm) method. Computers \& Fluids 37(4), 369-387.

[43] Ewert, R., J. Dierke, J. Siebert, A. Neifeld, C. Appel, M. Siefert, and O. Kornow (2011). Caa broadband noise prediction for aeroacoustic design. Journal of sound and vibration 330(17), 4139-4160.

[44] Hu, N., C. Appel, M. Herr, R. Ewert, and N. Reiche (2016). Numerical study of wall pressure fluctuations for zero and non-zero pressure gradient turbulent boundary layers. In 22nd AIAA/CEAS Aeroacoustics Conference, pp. 2911.

[45] Gravante, S. P., A. M. Naguib, C. E. Wark, and H. M. Nagib (1998). Characterization of the pressure fluctuations under a fully developed turbulent boundary layer. AIAA journal 36(10), 1808-1816.

[46] Tsuji, Y., S. Imayama, P. Schlatter, P. H. Alfredsson, A. V. Johansson, I. Marusic, N. Hutchins, and J. Monty (2012). Pressure fluctuation in high-reynolds-number turbulent boundary layer: results from experiments and dns. Journal of Turbulence (13), N50.

[47] Hu, N., C. Appel, M. Herr, R. Ewert, and N. Reiche (2016). Numerical study of wall pressure fluctuations for zero and non-zero pressure gradient turbulent boundary layers. In 22nd AIAA/CEAS Aeroacoustics Conference, pp. 2911.

[48] Howe, M. (1992). A note on the kraichnanâĂŤphillips theorem. Journal of Fluid Mechanics 234, 443-448.

[49] Howe, M. S. and M. S. Howe (1998). Acoustics of fluid-structure interactions. Cambridge university press.

[50] Shampine, L. F. (2008). Vectorized adaptive quadrature in matlab. Journal of Computational and Applied Mathematics 211(2), 131-140. 


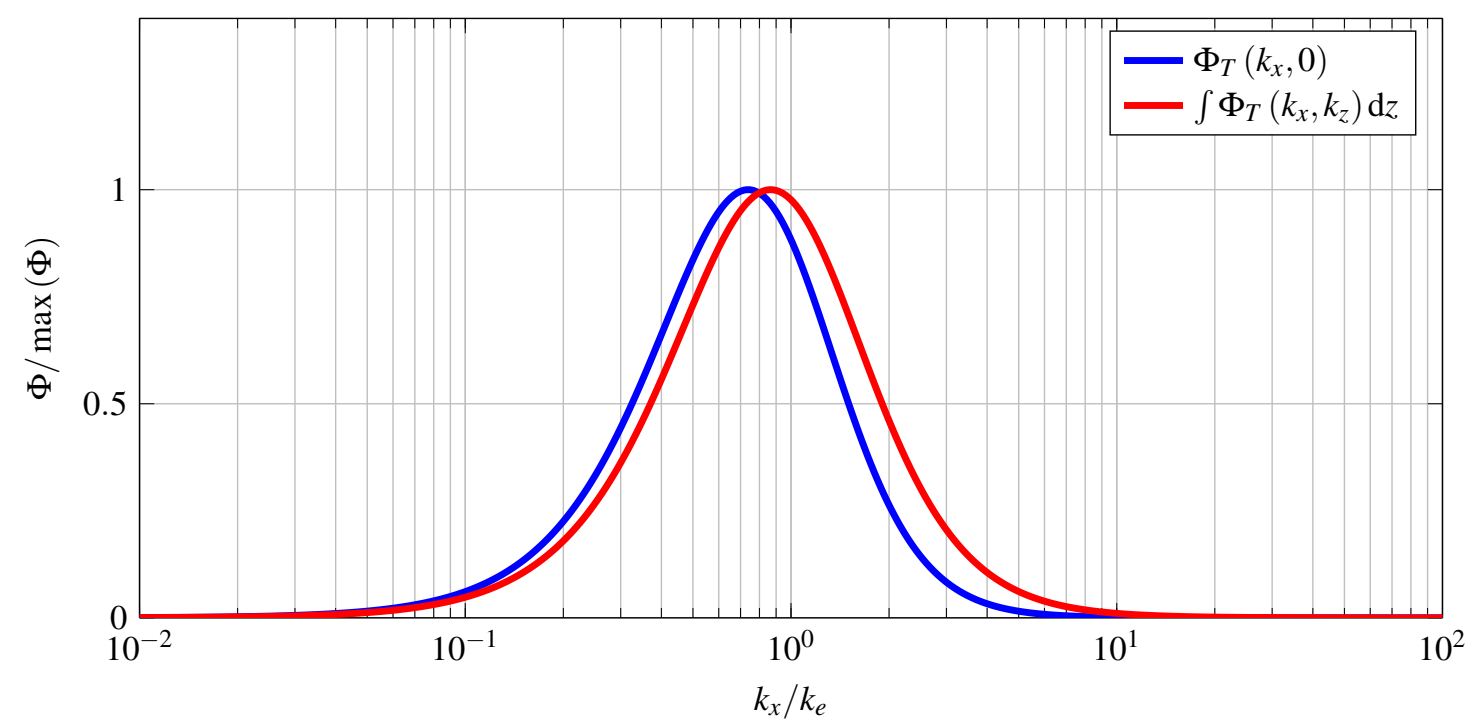

Fig. 1. Turbulence filters evolutions with respect to $k_{x}$ in a semilogarithmic plot

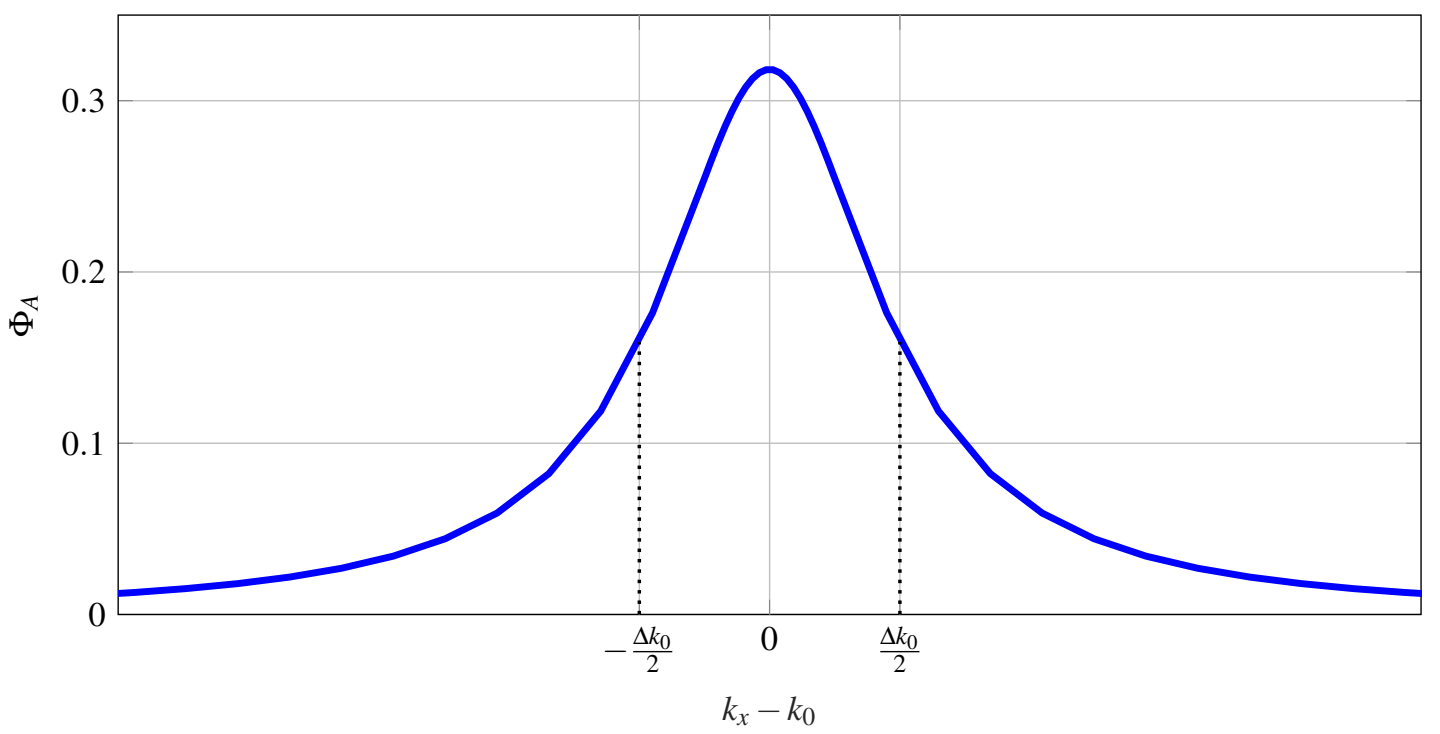

Fig. 2. Advection filter evolution with respect to $k_{x}$ 


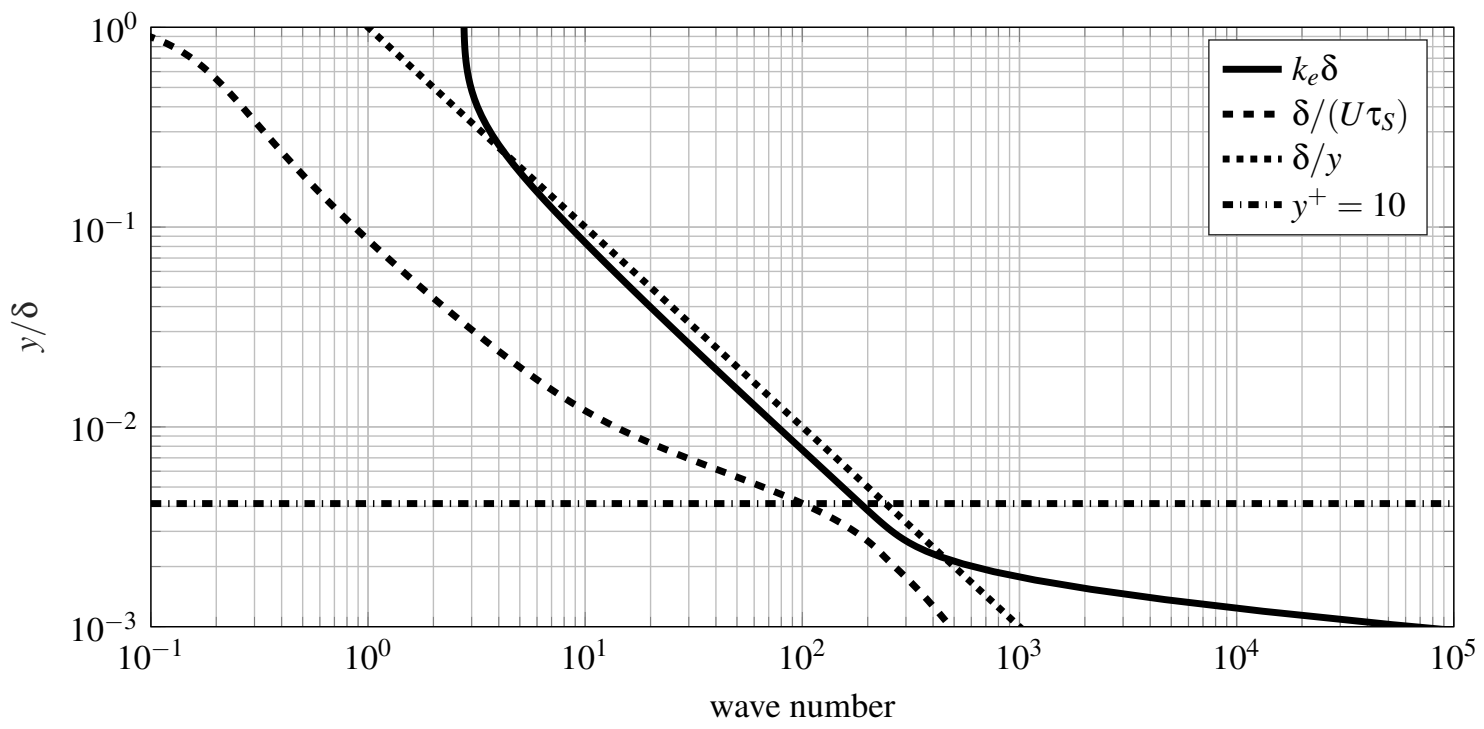

Fig. 3. Comparison of the bandwidths of the filters

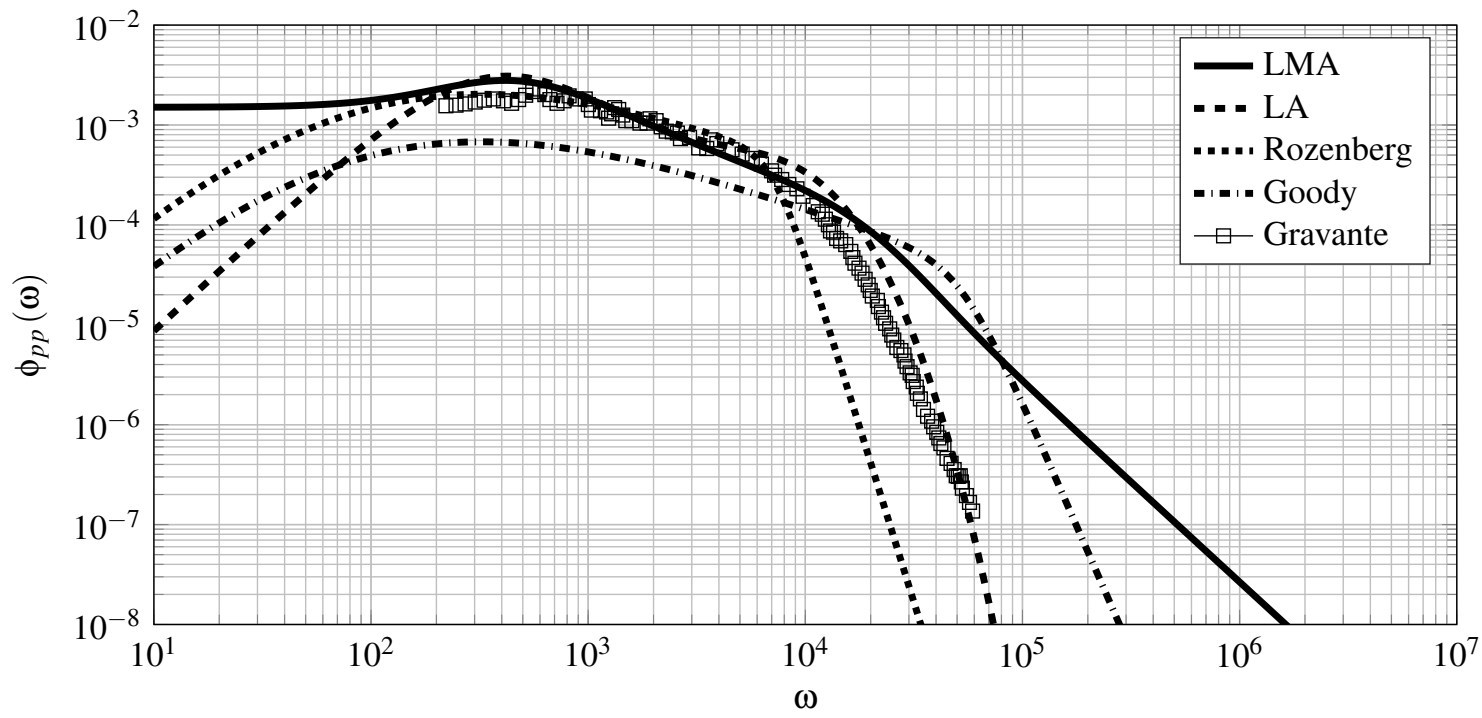

Fig. 4. Frequency dependency of $\phi_{p p}$ in a logarithmic representation

\begin{tabular}{|c|c|c|}
\hline & $k_{x} \rightarrow \Phi_{T}\left(k_{x}, 0\right)$ & $k_{x} \rightarrow \int \Phi_{T}\left(k_{x}, k_{z}\right) \mathrm{d} k_{z}$ \\
\hline \hline$k_{0}$ & $\approx 0.74 k_{e}$ & $\approx 0.87 k_{e}$ \\
\hline $\begin{array}{c}\text { Low cut-off } \\
-3 \mathrm{~dB}\end{array}$ & $\approx 0.42 k_{e}$ & $\approx 0.49 k_{e}$ \\
\hline $\begin{array}{c}\text { High cut-off } \\
-3 \mathrm{~dB}\end{array}$ & $\approx 1.22 k_{e}$ & $\approx 1.50 k_{e}$ \\
\hline $\begin{array}{c}\text { Bandwidth } \\
-3 \mathrm{~dB}\end{array}$ & $\approx 0.80 k_{e}$ & $\approx 1.01 k_{e}$ \\
\hline
\end{tabular}

Table 1. Band-widths of the turbulence filter 


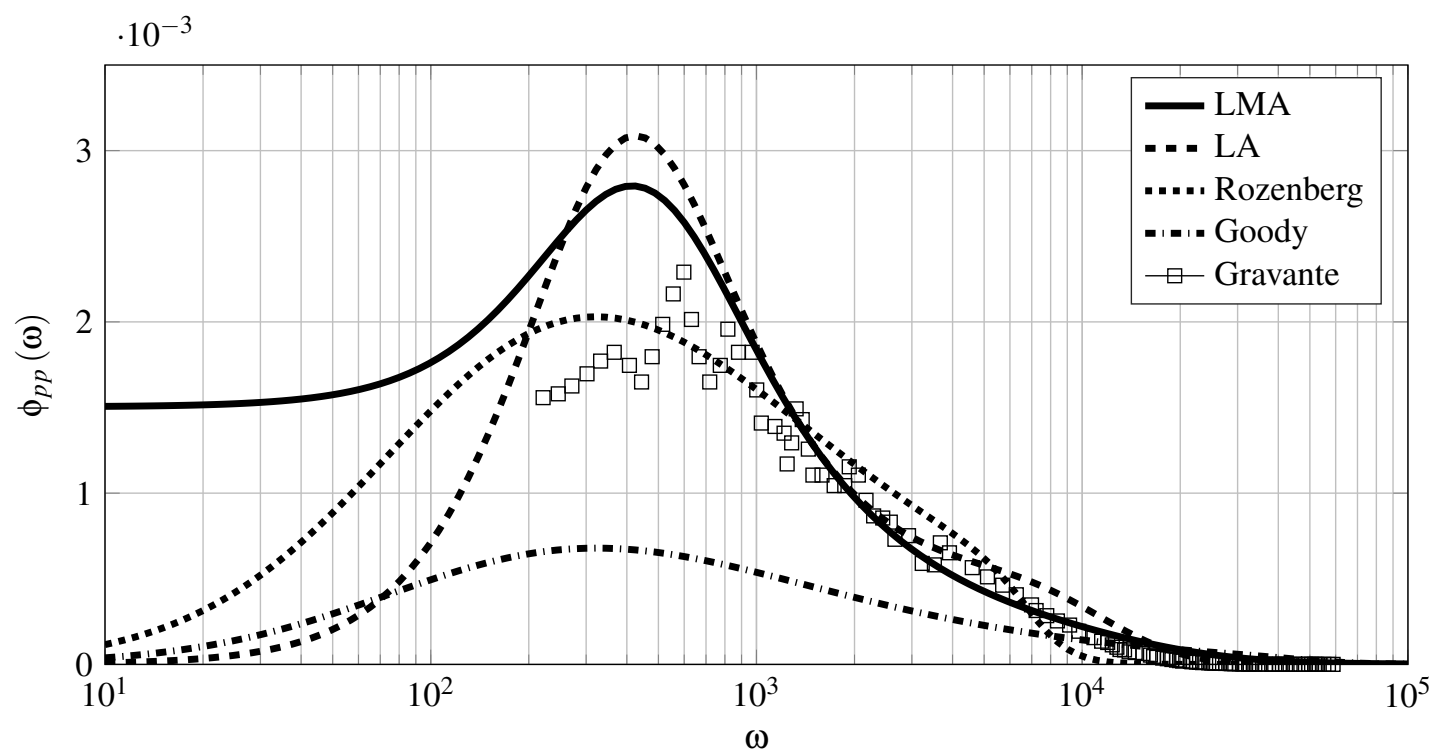

Fig. 5. Frequency dependency of $\phi_{p p}$ in a semi-logarithmic representation

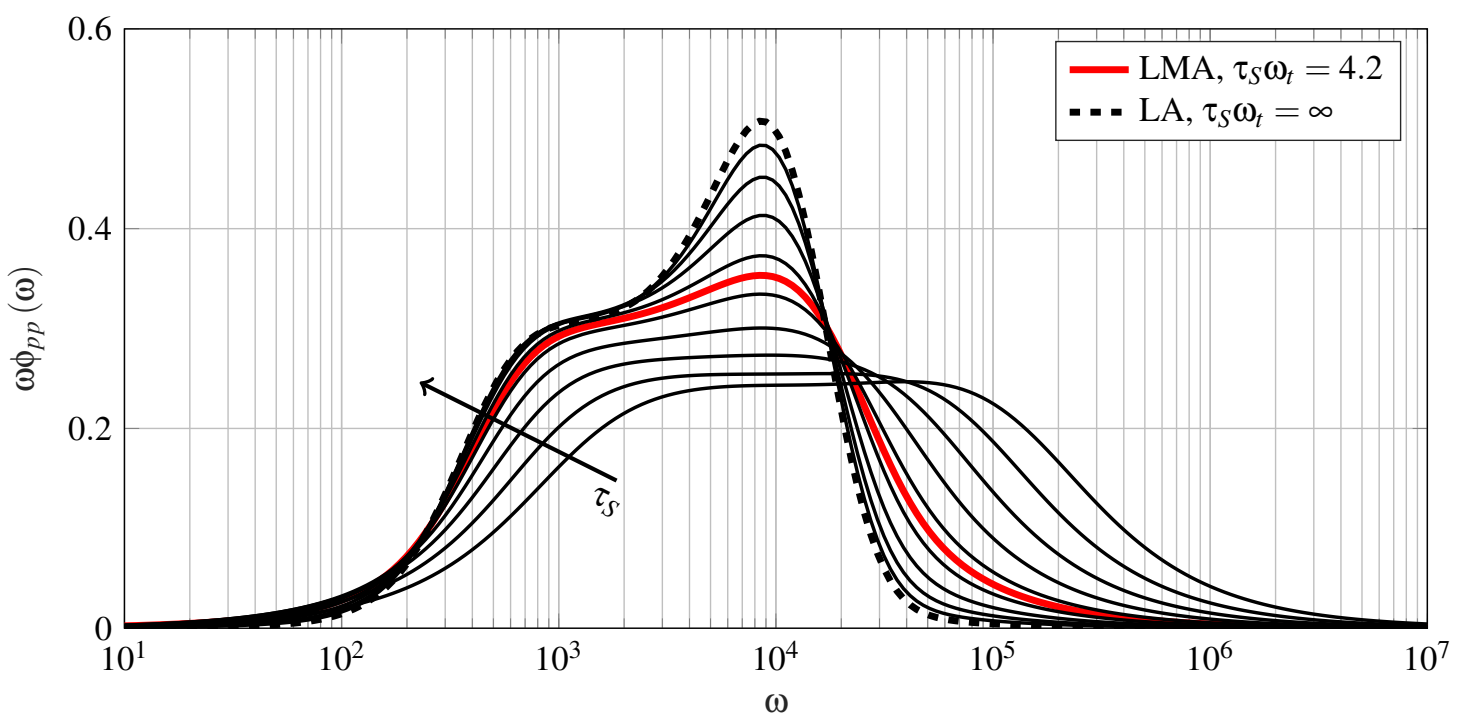

Fig. 6. Compensate frequency dependency of $\omega \phi_{p p}$ in a semi-logarithmic representation

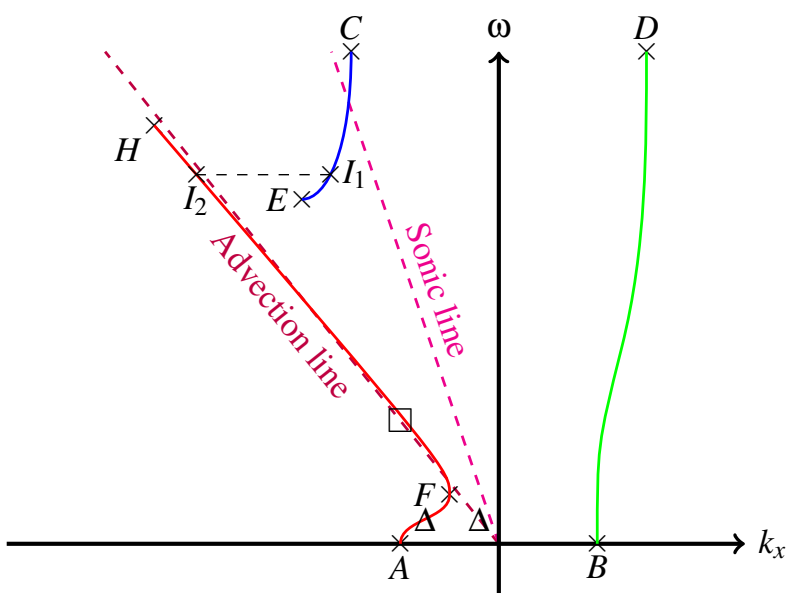

Fig. 7. Sketch of the three branch diagram 


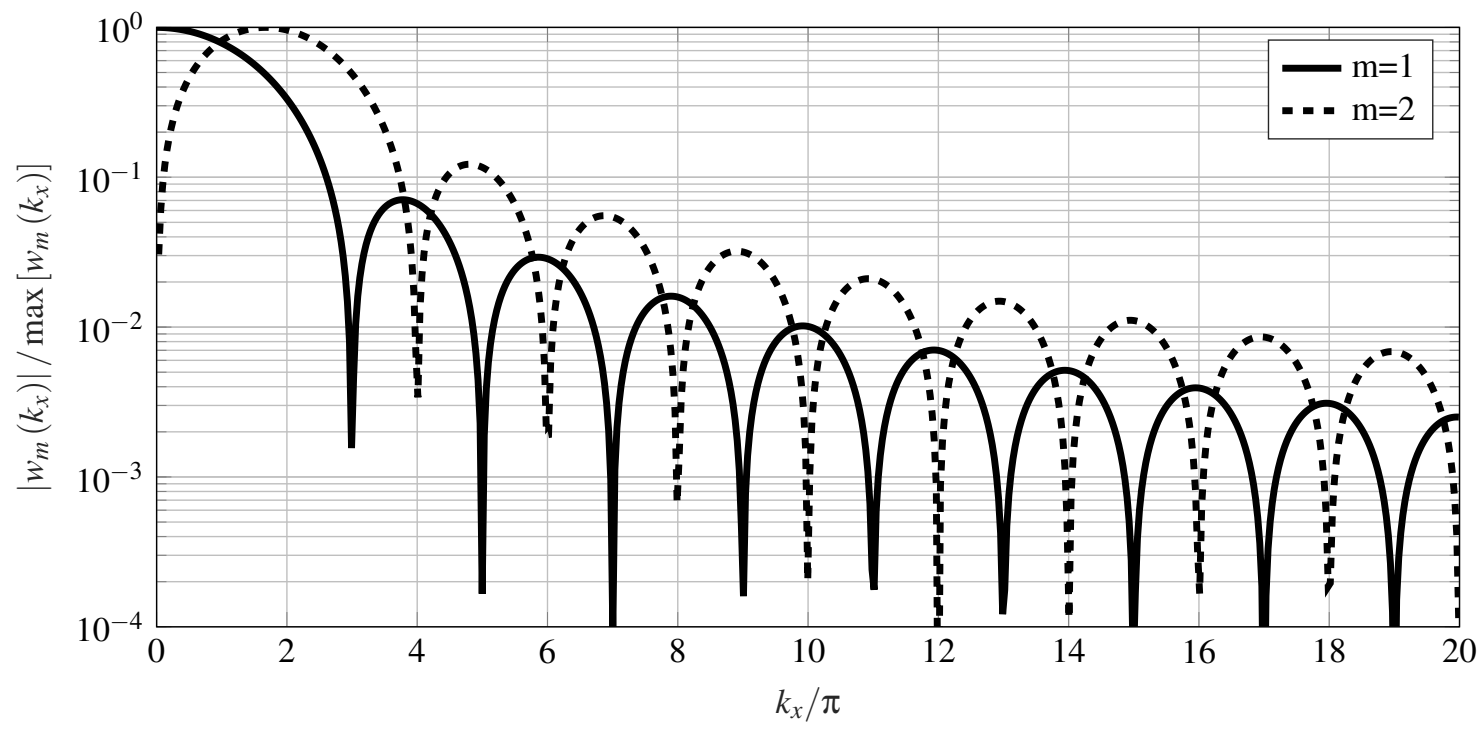

Fig. 8. Normalised modal function $w_{m n}$ evolution with respect to $k_{x}$

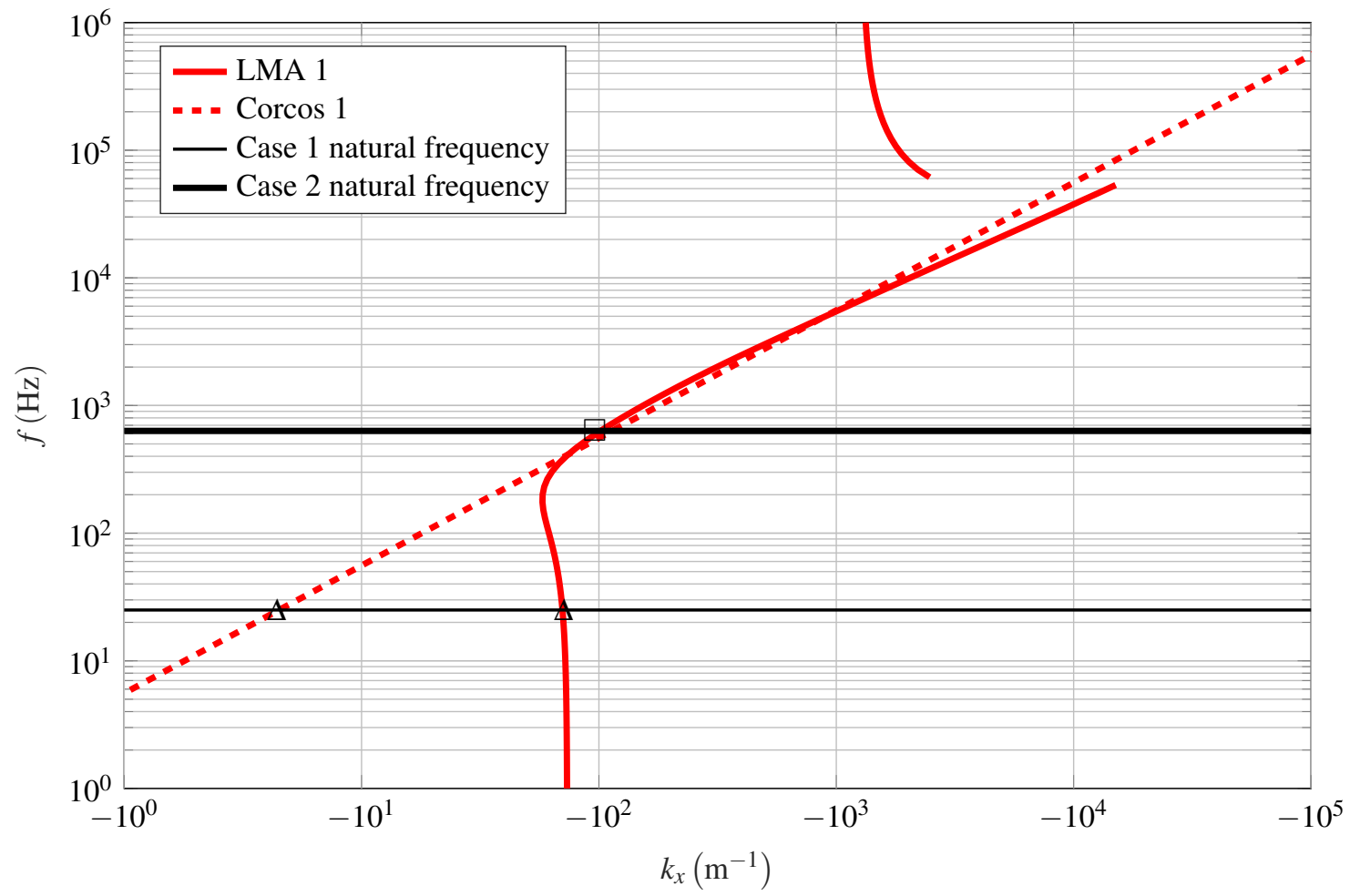

Fig. 9. Longitudinal dependency of $\Phi_{p p}(k, \omega)$ for a TBL with $U_{e}=50 \mathrm{~ms}^{-1}, R_{\theta}=3540$ 


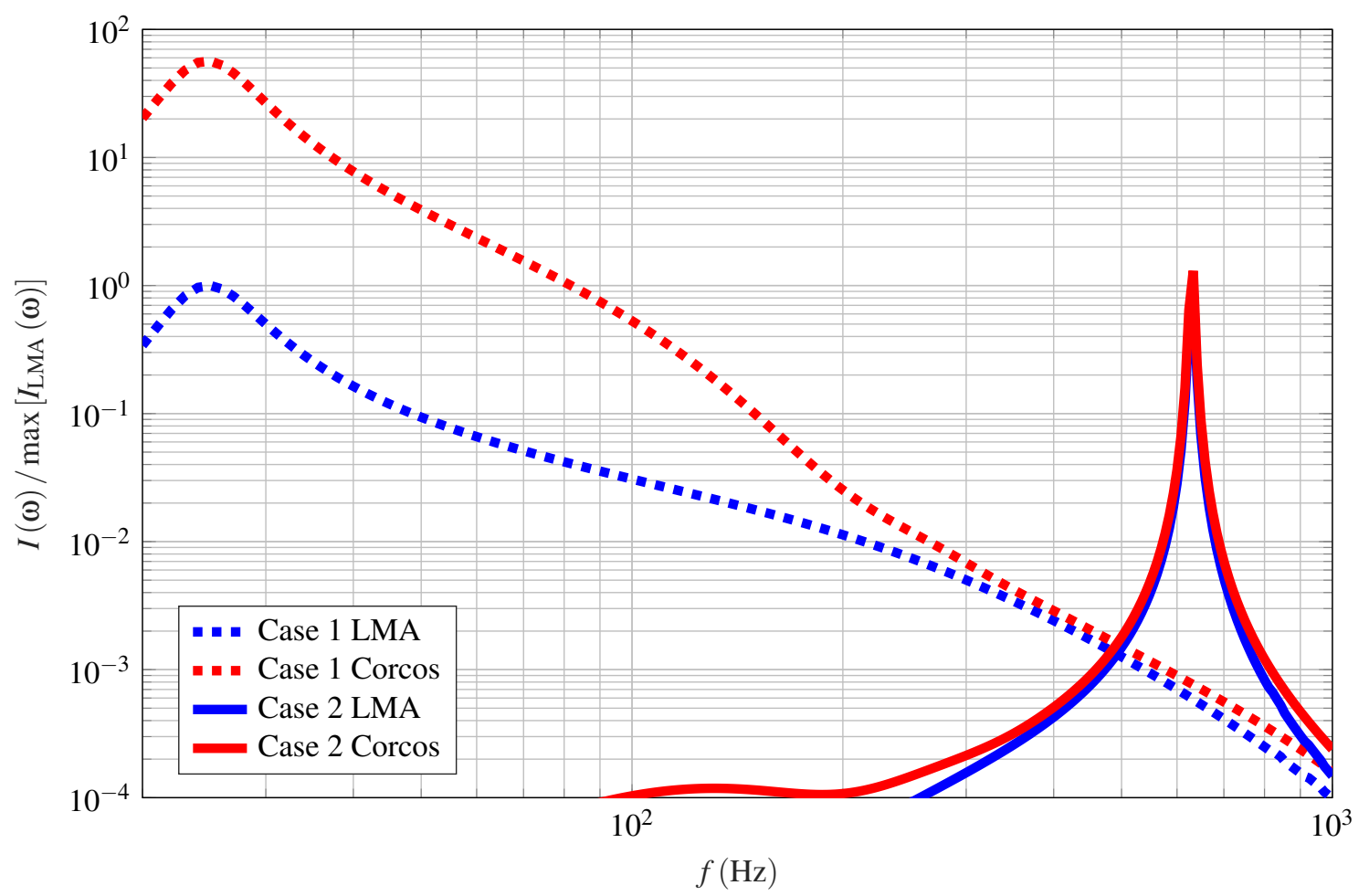

Fig. 10. Radiated noise by mode $(1,1)$

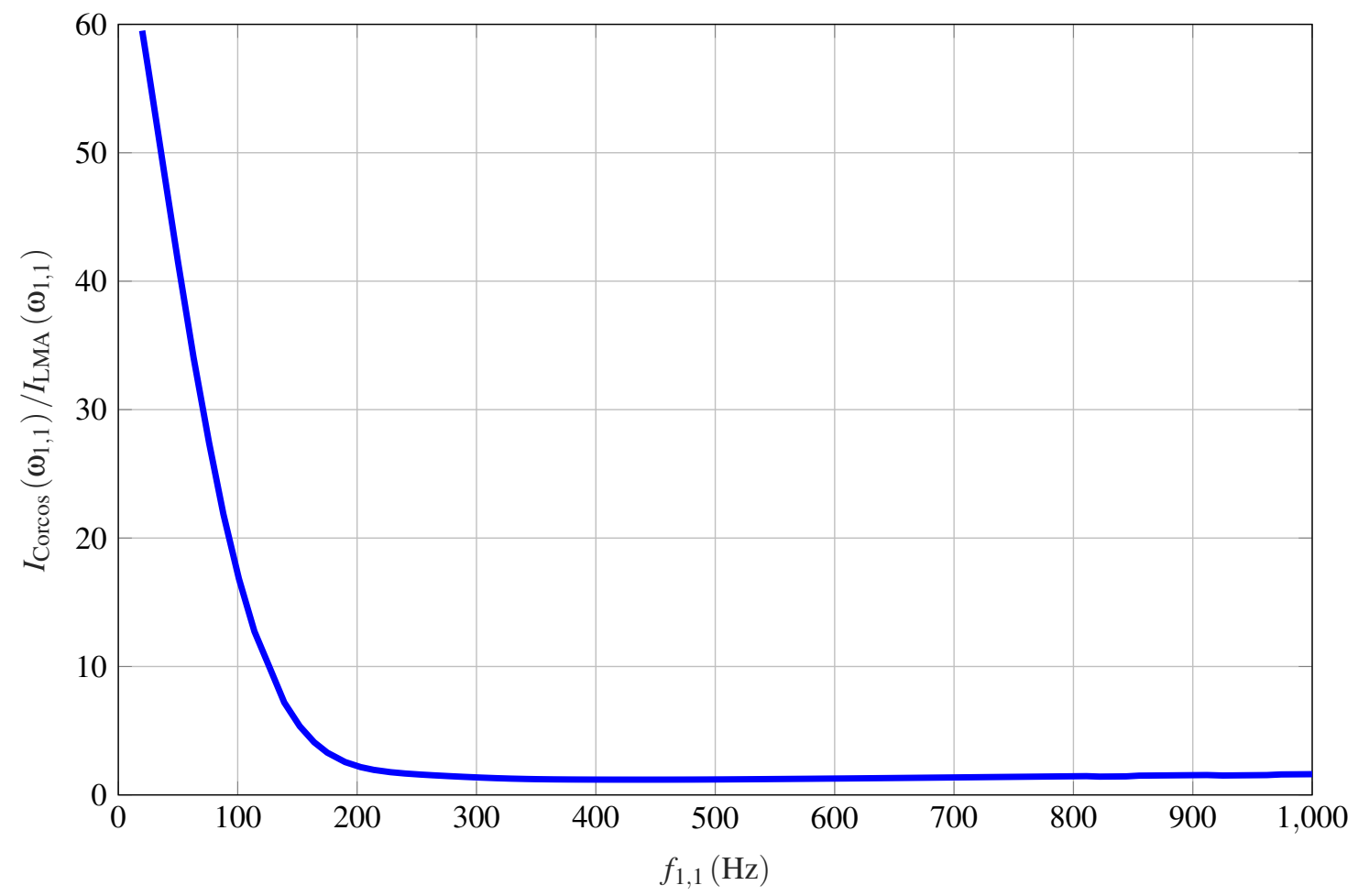

Fig. 11. Radiated noise by mode $(1,1)$ at the natural frequency 


\begin{tabular}{|c|c|c|c|c|}
\hline & & $A, B$ & $C, D$ & $E$ \\
\hline \hline \multirow{2}{*}{$R_{1 / k}=\beta_{k} R_{\theta}^{\alpha_{k}}$} & $\alpha_{k}$ & 1.1056 & 1.0487 & 1.0547 \\
\cline { 2 - 5 } & $\beta_{k}$ & 5.6485 & 3.2060 & 1.7399 \\
\hline \multirow{2}{*}{$R_{U / \omega}=\beta_{\omega} R_{\theta}^{\alpha_{\omega}}$} & $\alpha_{\omega}$ & & & 0.9977 \\
\cline { 2 - 5 } & $\beta_{\omega}$ & & & 1.2511 \\
\hline \hline & & $F$ & $I_{1}$ & \multicolumn{1}{|c|}{$I_{2}$} \\
\hline \hline \multirow{2}{*}{$R_{1 / k}=\beta_{k} R_{\theta}^{\alpha_{k}}$} & $\alpha_{k}$ & 1.1283 & 0.8184 & 1.0643 \\
\cline { 2 - 5 } & $\beta_{k}$ & 5.1988 & 1.1987 & 2.5771 \\
\hline \multirow{2}{*}{$R_{U / \omega}=\beta_{\omega} R_{\theta}^{\alpha_{\omega}}$} & $\alpha_{\omega}$ & 1.0759 & \multicolumn{2}{|c}{0.8103} \\
\cline { 2 - 5 } & $\beta_{\omega}$ & 21.3876 & \multicolumn{2}{|c}{2.1006} \\
\hline
\end{tabular}

Table 2. Correlations for characteristic points of the three branches diagram

\begin{tabular}{|c|c|c|}
\hline & Exact & Approximate \\
\hline \hline Frequency loop $\omega$ & 20000 & 20000 \\
\hline Modal loop $m, n$ & $100 \times 100$ & $\approx 100$ \\
\hline $\begin{array}{c}\text { Wave-number } \\
\text { integration loop } M, N\end{array}$ & $100 \times 100$ & $\approx 100$ \\
\hline $\begin{array}{c}\text { Boundary layer } \\
\text { integration loop } Y\end{array}$ & 150 & $\approx 50$ \\
\hline \hline Product & $3.10^{14}$ & $10^{10}$ \\
\hline Ratio & 1 & $1 / 30000$ \\
\hline
\end{tabular}

Table 3. Number of operations

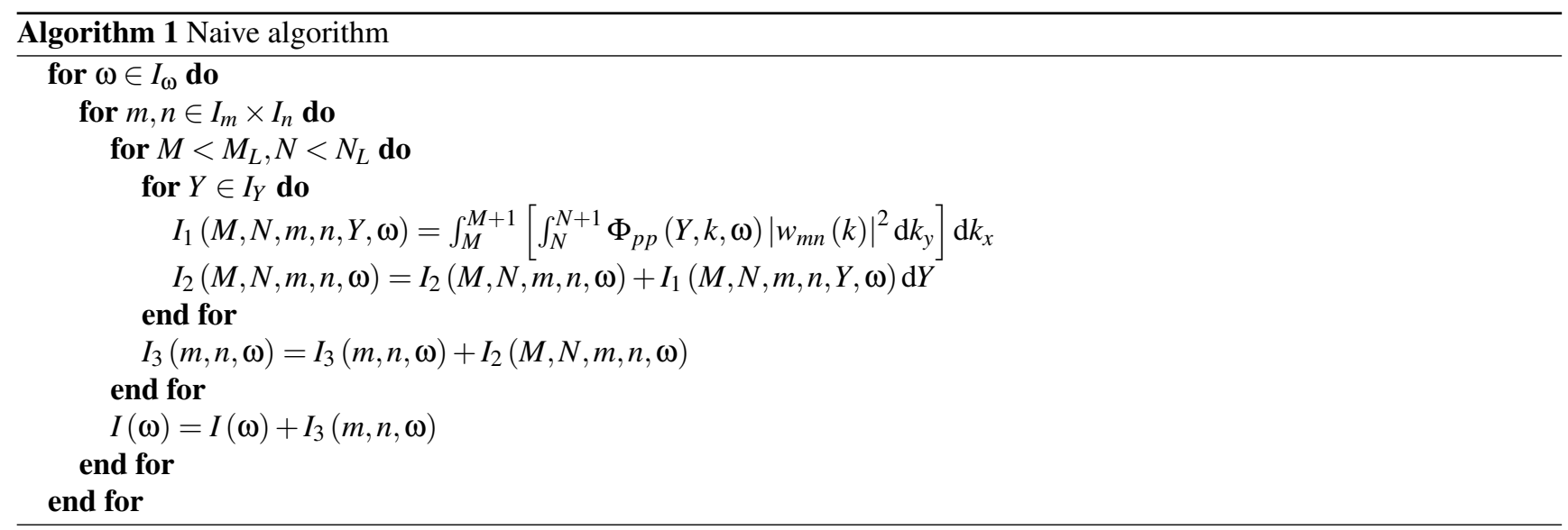




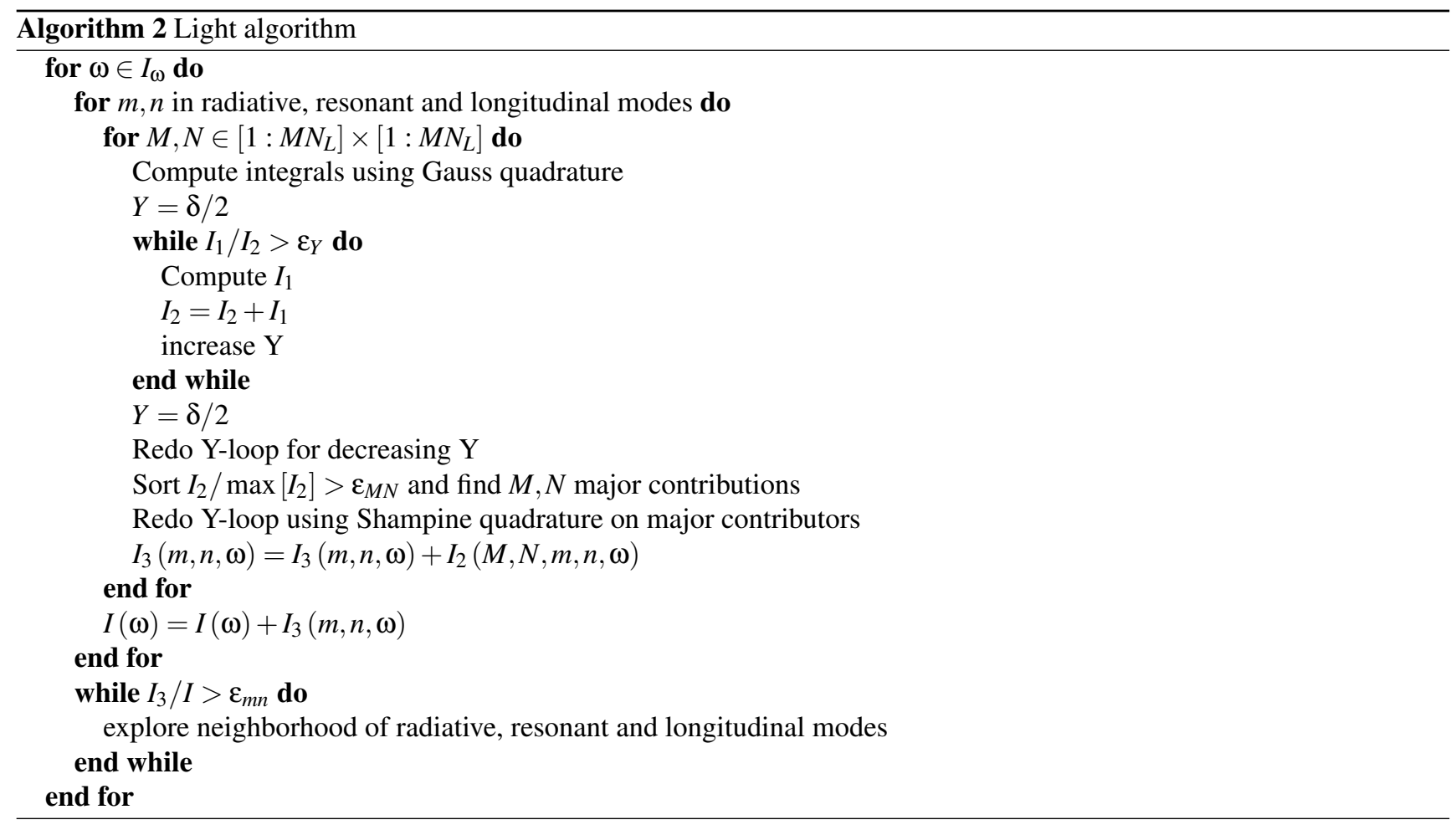

